\title{
A NEW KIND OF AUGMENTATION OF FILTRATIONS
}

\author{
Joseph NAJNUdel ${ }^{1}$ AND AshKAn Nikeghbali ${ }^{1}$
}

\begin{abstract}
Let $\left(\Omega, \mathcal{F},\left(\mathcal{F}_{t}\right)_{t>0}, \mathbb{P}\right)$ be a filtered probability space satisfying the usual assumptions: it is usually not possible to extend to $\mathcal{F}_{\infty}$ (the $\sigma$-algebra generated by $\left(\mathcal{F}_{t}\right)_{t \geq 0}$ ) a coherent family of probability measures $\left(\mathbb{Q}_{t}\right)$ indexed by $t \geq 0$, each of them being defined on $\mathcal{F}_{t}$. It is known that for instance, on the Wiener space, this extension problem has a positive answer if one takes the filtration generated by the coordinate process, made right-continuous, but can have a negative answer if one takes its usual augmentation. On the other hand, the usual assumptions are crucial in order to obtain the existence of regular versions of paths (typically adapted and continuous or adapted and càdlàg versions) for most stochastic processes of interest, such as the local time of the standard Brownian motion, stochastic integrals, etc. For instance we shall prove that on the Wiener space, equipped with the right-continuous augmentation of the filtration generated by the canonical process, there exists no càdlàg or continuous and adapted version for the local time at level zero of the canonical process. Hence there is an incompatibility between the problem of extending a coherent family of probability measures to $\mathcal{F}_{\infty}$ and the classical construction of regular versions of paths, requiring the usual assumptions (this situation typically occurs in the problem of penalization of the Brownian paths or in mathematical finance), which to the best of our knowledge, has not been noticed so far. In order to fix this problem, we introduce a new property for filtrations, intermediate between the right continuity and the usual conditions. More precisely, we say that a filtration $\left(\mathcal{F}_{t}\right)_{t \geq 0}$ satisfies the natural assumptions if it is right-continuous and if $\mathcal{F}_{0}$ contains all the sets included in a countable union of negligible sets $\left(B_{n}\right)_{n \geq 1}$, such that $B_{n} \in \mathcal{F}_{n}$ for $n \geq 1$. There is a natural way to obtain, from a given filtration $\left(\mathcal{F}_{t}\right)_{t \geq 0}$, a new filtration which satisfies the natural assumptions: we call it the natural augmentation of $\left(\mathcal{F}_{t}\right)_{t \geq 0}$. We show that most of the important results of the theory of stochastic processes which are generally proved under the usual augmentation, such as the existence of regular version of trajectories or the début theorem, still hold under the natural augmentation; moreover this new augmentation allows the extension of a coherent family of probability measures whenever this is possible with the original filtration. For sake of completeness, we also recall (not so well known) Parthasarathy type conditions on the underlying filtration under which the extension problem for a coherent family of probability measures has a solution. In particular, we shall see that this is always the case on the following two fundamental spaces: $\mathcal{C}\left(\mathbb{R}_{+}, \mathbb{R}\right)$, the space of continuous functions equipped with the filtration generated by the coordinate process and $\mathcal{D}\left(\mathbb{R}_{+}, \mathbb{R}\right)$, the space of càdlàg functions endowed with the filtration generated by the coordinate process.
\end{abstract}

Mathematics Subject Classification. 60G05, 60G07, 60G44.

Received December 11, 2009.

\footnotetext{
Keywords and phrases. The usual assumptions, change of probability measure.

${ }^{1}$ Institut für Mathematik, Universität Zürich, Winterthurerstrasse 190, 8057 Zürich, Switzerland.

joseph.najnudel@math.uzh.ch; ashkan.nikeghbali@math.uzh.ch
} 


\section{RECOGNITION OF PRIORITY}

After we put a preprint version of this paper on the arxiv, it was noticed by Ramon van Handel that the new augmentation we are proposing here was already introduced by Bichteler in 2002 in his book on stochastic integration [1]. Since the methods we are using are different and since some of our results are not contained in [1], we have decided to leave our original version unchanged (except for minor points) so that the reasons why we were led naturally to introduce this augmentation are still reflected in the present work.

\section{INTRODUCTION}

In stochastic analysis, most of the interesting properties of continuous time random processes cannot be established if one does not assume that their trajectories satisfy some regularity conditions. For example, a nonnegative càdlàg martingale converges almost surely, but if the càdlàg assumption is removed, the result becomes false in general. Recall a counter-example: on the filtered probability space $\left(\left(\mathcal{C}\left(\mathbb{R}_{+}, \mathbb{R}\right), \mathcal{F},\left(\mathcal{F}_{t}\right)_{t \geq 0}, \mathbb{W}\right)\right.$, where $\mathcal{F}_{t}=\sigma\left\{X_{s}, 0 \leq s \leq t\right\}, \mathcal{F}=\sigma\left\{X_{s}, s \geq 0\right\},\left(X_{s}\right)_{s \geq 0}$ is the canonical process and $\mathbb{W}$ the Wiener measure, the martingale

$$
\left(M_{t}:=\mathbb{1}_{X_{t}=1}\right)_{t \geq 0},
$$

which is a.s. equal to zero for each fixed $t \geq 0$, does not converge at infinity. That is the reason why one generally considers a càdlàg version of a martingale. However there are fundamental examples of stochastic processes for which such a version does not exist. Indeed let us define on the filtered probability space $\left(\left(\mathcal{C}\left(\mathbb{R}_{+}, \mathbb{R}\right), \mathcal{F},\left(\mathcal{F}_{t}\right)_{t \geq 0}, \mathbb{W}\right)\right.$ described above, the stochastic process $\left(\mathcal{L}_{t}\right)_{t \geq 0}$ as follows:

$$
\mathcal{L}_{t}=\Phi\left(\liminf _{m \rightarrow \infty} \int_{0}^{t} f_{m}\left(X_{s}\right) \mathrm{d} s\right)
$$

where $f_{m}$ denotes the density of a centered Gaussian variable with variance $1 / m$ and $\Phi$ is the function from $\mathbb{R}_{+} \cup\{\infty\}$ to $\mathbb{R}_{+}$such that $\Phi(x)=x$ for $x<\infty$ and $\Phi(\infty)=0$. The process $\left(\mathcal{L}_{t}\right)_{t \geq 0}$ is a version of the local time of the canonical process at level zero, which is defined everywhere and $\left(\mathcal{F}_{t}\right)_{t \geq 0}$-adapted. It is known that the process:

$$
\left(M_{t}:=\left|X_{t}\right|-\mathcal{L}_{t}\right)_{t \geq 0}
$$

is an $\left(\mathcal{F}_{t}\right)_{t>0}$-martingale. However, $\left(M_{t}\right)_{t>0}$ does not admit a càdlàg version which is adapted. In other words, there exists no càdlàg, adapted version $\left(\bar{L}_{t}\right)_{t \geq 0}$ for the local time at level zero of the canonical process! This property can be proved in the following way: let us consider an Ornstein-Uhlenbeck process $\left(U_{t}\right)_{t \geq 0}$, starting from zero, and let us define the process $\left(V_{t}\right)_{t \geq 0}$ by:

$$
V_{t}=(1-t) U_{t /(1-t)}
$$

for $t<1$, and

$$
V_{t}=0
$$

for $t \geq 1$. This process is a.s. continuous: we denote by $\mathbb{Q}$ its distribution. One can check the following properties:

- for all $t \in[0,1)$, the restriction of $\mathbb{Q}$ to $\mathcal{F}_{t}$ is absolutely continuous with respect to the corresponding restriction of $\mathbb{W}$;

- under $\mathbb{Q}, \mathcal{L}_{t} \rightarrow \infty$ a.s. when $t \rightarrow 1, t<1$.

By the second property, the set $\left\{\mathcal{L}_{t} \underset{t \rightarrow 1, t<1}{\longrightarrow} \infty\right\}$ has probability one under $\mathbb{Q}$. Since it is negligible under $\mathbb{P}$, it is essential to suppose that it is not contained in $\mathcal{F}_{0}$, if we need to have the first property: the filtration must 
not be completed. The two properties above imply

$$
\begin{aligned}
\mathbb{Q}\left[L_{1-2^{-n}} \underset{n \rightarrow \infty}{\longrightarrow} \infty\right] & \geq \mathbb{Q}\left[\mathcal{L}_{1-2^{-n}} \underset{n \rightarrow \infty}{\longrightarrow} \infty, \forall n \in \mathbb{N}, L_{1-2^{-n}}=\mathcal{L}_{1-2^{-n}}\right] \\
& \geq 1-\sum_{n \in \mathbb{N}} \mathbb{Q}\left[L_{1-2^{-n}} \neq \mathcal{L}_{1-2^{-n}}\right]=1 .
\end{aligned}
$$

The last equality is due to the fact that for all $n \in \mathbb{N}$,

$$
\mathbb{W}\left[L_{1-2^{-n}} \neq \mathcal{L}_{1-2^{-n}}\right]=0
$$

and then

$$
\mathbb{Q}\left[L_{1-2^{-n}} \neq \mathcal{L}_{1-2^{-n}}\right]=0
$$

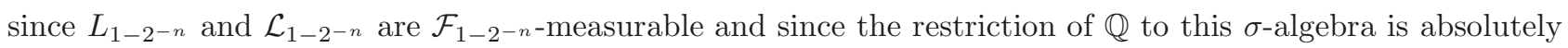
continuous with respect to $\mathbb{W}$. We have thus proved that there exist some paths such that $L_{1-2^{-n}}$ tends to infinity with $n$, which contradicts the fact that $\left(L_{t}\right)_{t>0}$ is càdlàg. From this we also deduce that in general there do not exist càdlàg versions for martingales. Similarly many other important results from stochastic analysis cannot be proved on the most general filtered probability space, e.g. the existence of the Doob-Meyer decomposition for submartinagales and the début theorem (see for instance [4] and [5]). In order to avoid this technical problem, it is generally assumed that the filtered probability space on which the processes are constructed satisfies the usual conditions, i.e. the filtration is complete and right-continuous.

But now, if we wish to perform a change of probability measure (for example, by using the Girsanov theorem), this assumption is too restrictive. Let us illustrate this fact by a simple example. Let us consider the filtered probability space $\left(\mathcal{C}\left(\mathbb{R}_{+}, \mathbb{R}\right), \widetilde{\mathcal{F}},\left(\widetilde{\mathcal{F}}_{t}\right)_{t \geq 0}, \widetilde{\mathbb{W}}\right)$ obtained, from the Wiener space $\left(\mathcal{C}\left(\mathbb{R}_{+}, \mathbb{R}\right), \mathcal{F},\left(\mathcal{F}_{t}\right)_{t \geq 0}, \mathbb{W}\right)$ described above, by taking its usual augmentation, i.e.:

- $\widetilde{\mathcal{F}}$ is the $\sigma$-algebra generated by $\mathcal{F}$ and its negligible sets;

- for all $t \geq 0, \widetilde{\mathcal{F}}_{t}$ is the $\sigma$-algebra generated by $\mathcal{F}_{t}$ and the negligible sets of $\mathcal{F}$;

- $\tilde{\mathbb{W}}$ is the unique possible extension of $\mathbb{W}$ to the completed $\sigma$-algebra $\widetilde{\mathcal{F}}$.

Let us also consider the family of probability measures $\left(\mathbb{Q}_{t}\right)_{t \geq 0}$, such that $\mathbb{Q}_{t}$ is defined on $\widetilde{\mathcal{F}}_{t}$ by:

$$
\mathbb{Q}_{t}=\mathrm{e}^{X_{t}-\frac{t}{2}} \cdot \widetilde{\mathbb{W}} \widetilde{\mathcal{F}}_{t}
$$

This family of probability measures is coherent, i.e. for $0 \leq s \leq t$, the restriction of $\mathbb{Q}_{t}$ to $\widetilde{\mathcal{F}}_{s}$ is equal to $\mathbb{Q}_{s}$. However, unlike what one would expect, there does not exist a probability measure $\mathbb{Q}$ on $\widetilde{\mathcal{F}}$ such that its restriction to $\widetilde{\mathcal{F}}_{s}$ is equal to $\mathbb{Q}_{s}$ for all $s \geq 0$. Indeed let us assume that $\mathbb{Q}$ exists. The event

$$
A:=\left\{\forall t \geq 0, X_{t} \geq-1\right\}
$$

satisfies $\widetilde{\mathbb{W}}[A]=0$, and then $A \in \widetilde{\mathcal{F}}_{0}$ by completeness, which implies that $\mathbb{Q}[A]=0$. On the other hand, under $\mathbb{Q}_{t}$, for all $t \geq 0$, the process $\left(X_{s}\right)_{0 \leq s \leq t}$ is a Brownian motion with drift 1 , and hence under $\mathbb{Q}$. One deduces that:

$$
\mathbb{Q}\left[\forall s \in[0, t], X_{s} \geq-1\right]=\widetilde{\mathbb{W}}\left[\forall s \in[0, t], X_{s} \geq-s-1\right] \geq \widetilde{\mathbb{W}}\left[\forall s \geq 0, X_{s} \geq-s-1\right] .
$$

Consequently by letting $t$ go to infinity one obtains:

$$
\mathbb{Q}[A] \geq \widetilde{\mathbb{W}}\left[\forall s \geq 0, X_{s} \geq-s-1\right]>0
$$

which is a contradiction. Therefore, the usual conditions are not suitable for the problem of extension of coherent probability measures. In fact one can observe that the argument above does not depend on the completeness of $\widetilde{\mathcal{F}}$, but only on the fact that $\widetilde{\mathcal{F}}_{0}$ contains all the sets in $\widetilde{\mathcal{F}}$ of probability zero. That is why it still remains 
available if we consider, with the notation above, the space $\left(\mathcal{C}\left(\mathbb{R}_{+}, \mathbb{R}\right), \mathcal{F},\left(\mathcal{F}_{t}^{\prime}\right)_{t \geq 0}, \mathbb{W}\right)$, where for all $t \geq 0, \mathcal{F}_{t}^{\prime}$ is the $\sigma$-algebra generated by $\mathcal{F}_{t}$ and the sets in $\mathcal{F}$ of probability zero.

In order to illustrate our point, we now show that if one is not careful with the completion, then one can easily obtain an extension of the Lebesgue measure to all subsets of the real line. Indeed, assume that on the measurable space $(\Omega, \widetilde{\mathcal{F}})$ (without any filtration), there exists a probability measure under which the canonical process is a Brownian motion with drift one. Since the event $\left\{X_{t} \underset{t \rightarrow \infty}{\longrightarrow} \infty\right\}$ is in $\mathcal{F}$ and has probability zero under the Wiener measure, all its subsets are contained in $\widetilde{\mathcal{F}}$. Then, if under a probability $\mathbb{Q},\left(X_{t}\right)_{t \geq 0}$ is a Brownian motion with drift one, one can define a finite measure $\mu$ on $\mathcal{P}([0,1])$ by:

$$
\mu(A)=\mathbb{Q}\left[F\left(X_{1}-1\right) \in A, X_{t} \underset{t \rightarrow \infty}{\longrightarrow} \infty\right]
$$

for all subsets $A$ of $[0,1]$, where $F$ is the distribution function of the standard Gaussian variable. Since under $\mathbb{Q}, X_{t}$ tends almost surely to infinity when $t \rightarrow \infty$, one checks that $\mu$ is an extension of the Lebesgue measure, which is defined for all the subsets of $[0,1]$. Now, the existence of such an extension is incompatible with the continuum hypothesis if one assumes the usual axioms of set theory (see [2] for details and references on this problem).

The above discussion (which corresponds to the simplest non-trivial Girsanov transformation) outlines that by doing the usual augmentation, we add, in a certain sense, too much information in the filtration which is considered: in particular the asymptotic properties of the processes are put directly into $\mathcal{F}_{0}$. It is precisely the fact that asymptotic events are already in $\mathcal{F}_{0}$, which implies that it is not possible in general to construct a probability measure $\mathbb{Q}$ which is singular with respect to $\mathbb{W}$ but locally absolutely continuous with respect to it. Let us sum up the two problems we have encountered:

- if we do not complete the filtrations, most of the properties of the trajectories of the stochastic processes are lost in general;

- if we add all the negligible sets in $\mathcal{F}_{0}$, it is not possible in general to extend to $\mathcal{F}_{\infty}=\sigma\left(\bigcup_{t>0} \mathcal{F}_{t}\right)$ a coherent family of probability measures defined on $\mathcal{F}_{t}$. In particular, it is not possible to construct, by Girsanov transformation, a probability which is singular with respect to the initial probability measure.

Now a natural question is: what can one do to avoid simultaneously these two issues? This case arises, for instance, if one needs to perform Giranov transformations with a density involving local times. It also occurs when, working with an infinite time horizon, one defines locally on each $\mathcal{F}_{t}$ a new probability measure with the help of a martingale which is not uniformly integrable (e.g. $\exp \left(\gamma X_{t}-\gamma^{2} t / 2\right)$ on the Wiener space) and then one manipulates stochastic integrals, local times, etc. This situation is very often encountered in some problems related to the penalization of the Wiener paths or sometimes in financial modeling. To the best of our knowledge this important issue has never been dealt with before, hence making the results obtained when facing these two problems not totally rigorous.

The goal of this paper is to show that by performing a new kind of augmentation of filtrations, intermediate between the right-continuous version and the usual augmentation, it is possible to address the two technical issues addressed above. This augmentation, called natural augmentation, is obtained as follows: after making the filtration right-continuous, instead of putting all the negligible sets of $\mathcal{F}$ in $\mathcal{F}_{0}$ (which adds too much information), we only put the sets which are contained in a countable union $\left(B_{n}\right)_{n \geq 0}$ of sets of probability zero, such that $B_{n} \in \mathcal{F}_{n}$ for all $n \geq 0$. Note that this way of completing filtrations has a concrete interpretation, which can be interesting in modeling problems: the events of probability zero can be anticipated if they concern the future only up to a finite time, even if this time is unbounded.

More precisely the paper is organized as follows:

- in Section 2, we construct in detail the natural augmentation of a filtration and compare it with the usual augmentation;

- in Section 3, we show that most of the classical theorems of stochastic analysis which are proved under the usual conditions remain true under natural conditions; 
- in Section 4 we show that the natural augmentation preserves the main properties of the stochastic processes (martingale property for example), and allows us to deal with measures which are singular with respect to the initial probability measure. In particular, we review sufficient conditions of Parthasarathy type under which a coherent family of probability measures can be extended, and we show that in the most interesting cases, this extension remains possible after taking the natural augmentation.

\section{The natural augmentation of Filtrations}

Let $\left(\Omega, \mathcal{F},\left(\mathcal{F}_{t}\right)_{t \geq 0}, \mathbb{P}\right)$ be a filtered probability space. We first introduce a few definitions in order to rigorously define the natural conditions. The first notion we introduce is the notion of $\sigma$-negligible sets, which corresponds to the sets we want to put into $\mathcal{F}_{0}$ in the natural augmentation.

Definition 2.1. A subset $A$ of $\Omega$ is $\sigma$-negligible with respect to the space $\left(\Omega, \mathcal{F},\left(\mathcal{F}_{t}\right)_{t \geq 0}, \mathbb{P}\right)$, if and only if there exists a sequence $\left(B_{n}\right)_{n \geq 0}$ of subsets of $\Omega$, such that for all $n \geq 0, B_{n} \in \mathcal{F}_{n}, \mathbb{P}\left[B_{n}\right]=0$, and

$$
A \subset \bigcup_{n \geq 0} B_{n} .
$$

Remark 2.2. The integers do not play a crucial rôle in Definition 2.1. If $\left(t_{n}\right)_{n \geq 0}$ is an unbounded sequence in $\mathbb{R}_{+}$, one can replace the condition $B_{n} \in \mathcal{F}_{n}$ by the condition $B_{n} \in \mathcal{F}_{t_{n}}$.

Let us now define a notion which is the analog of completeness for $\sigma$-negligible sets. It is the main ingredient in the definition of what we shall call the natural conditions:

Definition 2.3. A filtered probability space $\left(\Omega, \mathcal{F},\left(\mathcal{F}_{t}\right)_{t \geq 0}, \mathbb{P}\right)$, is $\sigma$-complete if and only if all the $\sigma$-negligible sets of this space are contained in $\mathcal{F}_{0}$. It satisfies the natural conditions if and only if it is $\sigma$-complete and the filtration $\left(\mathcal{F}_{t}\right)_{t \geq 0}$ is right-continuous.

It is natural to ask if from a given filtered probability space, one can define in a canonical way a space which satisfies the natural conditions and which is as "close" as possible to the initial space. The answer to this question is positive in the following sense:

Proposition 2.4. Let $\left(\Omega, \mathcal{F},\left(\mathcal{F}_{t}\right)_{t \geq 0}, \mathbb{P}\right)$ be a filtered probability space, and $\mathcal{N}$ the family of its $\sigma$-negligible sets. Let $\widetilde{\mathcal{F}}$ be the $\sigma$-algebra generated by $\mathcal{N}$ and $\mathcal{F}$, and for all $t \geq 0, \widetilde{\mathcal{F}}_{t}$ the $\sigma$-algebra generated by $\mathcal{N}$ and $\mathcal{F}_{t+}$, where

$$
\mathcal{F}_{t+}:=\bigcap_{u>t} \mathcal{F}_{u} .
$$

Then there exists a unique probability measure $\widetilde{\mathbb{P}}$ on $(\Omega, \widetilde{\mathcal{F}})$ which coincides with $\mathbb{P}$ on $\mathcal{F}$, and the space $\left(\Omega, \widetilde{\mathcal{F}},\left(\widetilde{\mathcal{F}}_{t}\right)_{t \geq 0}, \widetilde{\mathbb{P}}\right)$ satisfies the natural conditions. Moreover, if $\left(\Omega, \mathcal{F}^{\prime},\left(\mathcal{F}_{t}^{\prime}\right)_{t \geq 0}, \mathbb{P}^{\prime}\right)$ is a filtered probability space satisfying the natural conditions, such that $\mathcal{F}^{\prime}$ contains $\mathcal{F}, \mathcal{F}_{t}^{\prime}$ contains $\mathcal{F}_{t}$ for all $t \geq 0$, and if $\mathbb{P}^{\prime}$ is an extension of $\mathbb{P}$, then $\mathcal{F}^{\prime}$ contains $\widetilde{\mathcal{F}}, \mathcal{F}^{\prime}$ contains $\widetilde{\mathcal{F}}_{t}$, for all $t \geq 0$ and $\mathbb{P}^{\prime}$ is an extension of $\widetilde{\mathbb{P}}$. In other words, $\left(\Omega, \widetilde{\mathcal{F}},\left(\widetilde{\mathcal{F}}_{t}\right)_{t \geq 0}, \widetilde{\mathbb{P}}\right)$ is the smallest extension of $\left(\Omega, \mathcal{F},\left(\mathcal{F}_{t}\right)_{t \geq 0}, \mathbb{P}\right)$ which satisfies the natural conditions: we call it the natural augmentation of $\left(\Omega, \mathcal{F},\left(\mathcal{F}_{t}\right)_{t \geq 0}, \mathbb{P}\right)$.

Proof. Let us first denote by $\mathcal{E}$ the family of subsets $A$ of $\Omega$ such that there exists $A^{\prime} \in \mathcal{F}$, satisfying:

$$
\left(A \backslash A^{\prime}\right) \cup\left(A^{\prime} \backslash A\right) \in \mathcal{N} .
$$

If $A \in \mathcal{E}$, if $A^{\prime}$ satisfies (2.1), and if we denote by $B$ the complement of $A, B^{\prime}$ the complement of $A^{\prime}$, then $B^{\prime} \in \mathcal{F}$ and:

$$
\left(B \backslash B^{\prime}\right) \cup\left(B^{\prime} \backslash B\right)=\left(A^{\prime} \backslash A\right) \cup\left(A \backslash A^{\prime}\right) \in \mathcal{N},
$$


which implies that $B \in \mathcal{E}$. Moreover, if $A_{n} \in \mathcal{E}$ for all $n \geq 1$, then there exists, for all $n \geq 1, A_{n}^{\prime} \in \mathcal{F}$ such that:

$$
\left(A_{n} \backslash A_{n}^{\prime}\right) \cup\left(A_{n}^{\prime} \backslash A_{n}\right) \in \mathcal{N} .
$$

One has:

$$
\left[\left(\bigcup_{n \geq 1} A_{n}\right) \backslash\left(\bigcup_{n \geq 1} A_{n}^{\prime}\right)\right] \cup\left[\left(\bigcup_{n \geq 1} A_{n}^{\prime}\right) \backslash\left(\bigcup_{n \geq 1} A_{n}\right)\right] \subset \bigcup_{n \geq 1}\left[\left(A_{n} \backslash A_{n}^{\prime}\right) \cup\left(A_{n}^{\prime} \backslash A_{n}\right)\right]
$$

and then,

$$
\left[\left(\bigcup_{n \geq 1} A_{n}\right) \backslash\left(\bigcup_{n \geq 1} A_{n}^{\prime}\right)\right] \cup\left[\left(\bigcup_{n \geq 1} A_{n}^{\prime}\right) \backslash\left(\bigcup_{n \geq 1} A_{n}\right)\right] \in \mathcal{N}
$$

since $\mathcal{N}$ is stable by countable union. Therefore,

$$
\bigcup_{n \geq 1} A_{n} \in \mathcal{E}
$$

and $\mathcal{E}$ is a $\sigma$-algebra. Since it obviously contains $\mathcal{F}$ and $\mathcal{N}$, it contains $\widetilde{\mathcal{F}}$. On the other hand, if $A \in \mathcal{E}$, and $A^{\prime}$ satisfies (2.1), then:

$$
A=\left[A^{\prime} \cup\left(A \backslash A^{\prime}\right)\right] \backslash\left(A^{\prime} \backslash A\right) \in \widetilde{\mathcal{F}},
$$

since $A^{\prime} \in \mathcal{F}, A \backslash A^{\prime} \in \mathcal{N}$ and $A^{\prime} \backslash A \in \mathcal{N}$. In other words, we have proved:

$$
\mathcal{E}=\widetilde{\mathcal{F}} .
$$

Similarly, for all $t \geq 0$, a set $A$ is in $\widetilde{\mathcal{F}}_{t}$ if and only if there exists $A^{\prime} \in \mathcal{F}_{t+}$ satisfying (2.1). Now, let $\widetilde{\mathbb{P}}$ be a probability on $(\Omega, \widetilde{\widetilde{\mathcal{F}}})$ extending $\mathbb{P}$. If $A \in \widetilde{\mathcal{F}}$, and $A^{\prime} \in \mathcal{F}$ satisfies $(2.1)$, then

$$
\widetilde{\mathbb{P}}\left[A \backslash A^{\prime}\right]=\widetilde{\mathbb{P}}\left[A^{\prime} \backslash A\right]=0,
$$

since these two sets, $\sigma$-negligible, are included in a set in $\mathcal{F}$ of probability zero. This implies:

$$
\widetilde{\mathbb{P}}[A]=\mathbb{P}\left[A^{\prime}\right]+\widetilde{\mathbb{P}}\left[A \backslash A^{\prime}\right]-\widetilde{\mathbb{P}}\left[A^{\prime} \backslash A\right]=\mathbb{P}\left[A^{\prime}\right]
$$

and uniqueness of $\widetilde{\mathbb{P}}$ if it exists. To prove existence, let us first observe that for three sets $A \in \widetilde{\mathcal{F}}, A^{\prime}, A^{\prime \prime} \in \mathcal{F}$ satisfying (2.1) and the similar equation with $A^{\prime}$ replaced by $A^{\prime \prime}$,

$$
\mathbb{P}\left[A^{\prime}\right]=\mathbb{P}\left[A^{\prime \prime}\right] .
$$

This is a consequence of the fact that $A^{\prime} \backslash A^{\prime \prime}$ and $A^{\prime \prime} \backslash A^{\prime}$ are in the intersection of $\mathcal{N}$ and $\mathcal{F}$, and then, have probability zero. In other words, if $A \in \widetilde{\mathcal{F}}$, one can define:

$$
\widetilde{\mathbb{P}}[A]:=\mathbb{P}\left[A^{\prime}\right],
$$

since $\mathbb{P}\left[A^{\prime}\right]$ does not depend of the choice of $A^{\prime}$ satisfying (2.1). Since for $A \in \mathcal{F}$, one can take $A^{\prime}=A$, $\widetilde{\mathbb{P}}$ is an extension of $\mathbb{P}$. Now let $\left(A_{n}\right)_{n \geq 1}$ be a sequence of disjoint sets in $\widetilde{\mathcal{F}}$, and $\left(A_{n}^{\prime}\right)_{n \geq 1}$ a sequence of sets in $\mathcal{F}$ satisfying the analog of (2.1). One has

$$
\left[\left(\bigcup_{n \geq 1} A_{n}\right) \backslash\left(\bigcup_{n \geq 1} A_{n}^{\prime}\right)\right] \cup\left[\left(\bigcup_{n \geq 1} A_{n}^{\prime}\right) \backslash\left(\bigcup_{n \geq 1} A_{n}\right)\right] \in \mathcal{N}
$$


and then

$$
\widetilde{\mathbb{P}}\left[\bigcup_{n \geq 1} A_{n}\right]=\mathbb{P}\left[\bigcup_{n \geq 1} A_{n}^{\prime}\right] .
$$

Now for $1 \leq m \leq n$,

$$
\mathbb{P}\left[A_{m}^{\prime} \cap A_{n}^{\prime}\right]=0,
$$

since $A_{m}$ and $A_{n}$ are disjoint, and then

$$
\widetilde{\mathbb{P}}\left[\bigcup_{n \geq 1} A_{n}\right]=\sum_{n \geq 1} \mathbb{P}\left[A_{n}^{\prime}\right]=\sum_{n \geq 1} \widetilde{\mathbb{P}}\left[A_{n}\right]
$$

which implies that $\widetilde{\mathbb{P}}$ is a probability measure. Let us now prove that $\left(\Omega, \widetilde{\mathcal{F}},\left(\widetilde{\mathcal{F}}_{t}\right)_{t \geq 0}, \widetilde{\mathbb{P}}\right)$ satisfies the natural conditions. The $\sigma$-completeness can be checked in the following way. If $A$ is an $\sigma$-negligible set with respect to $\left(\Omega, \widetilde{\mathcal{F}},\left(\widetilde{\mathcal{F}}_{t}\right)_{t \geq 0}, \widetilde{\mathbb{P}}\right)$, there exists $\left(B_{n}\right)_{n \geq 1}$ such that $B_{n} \in \widetilde{\mathcal{F}}_{n}$ and $\widetilde{\mathbb{P}}\left[B_{n}\right]=0$ for all $n \geq 1$, and:

$$
A \subset \bigcup_{n \geq 1} B_{n}
$$

Since $B_{n} \in \widetilde{\mathcal{F}}_{n}$ there exists $B_{n}^{\prime} \in \mathcal{F}_{n+}$ satisfying the analog of (2.1), which implies

$$
\mathbb{P}\left[B_{n}^{\prime}\right]=\widetilde{\mathbb{P}}\left[B_{n}\right]=0
$$

and then $B_{n}^{\prime} \in \mathcal{N}$. Since $B_{n} \backslash B_{n}^{\prime} \in \mathcal{N}, B_{n} \cup B_{n}^{\prime}$ and then $B_{n}$ are in $\mathcal{N}$. Finally the union of $B_{n}$ for $n \geq 1$ is also in $\mathcal{N}$, which implies $A \in \mathcal{N}^{n}$ and then $A \in \widetilde{\mathcal{F}}_{0}$. Let us now prove the right-continuity of $\left(\widetilde{\mathcal{F}}_{t}\right)_{t \geq 0}$. Let $t \geq 0$ and let $A$ be in the intersection of $\widetilde{\mathcal{F}}_{s}$ for $s>t$. For all integers $n \geq 1$ there exists $A_{n}^{\prime} \in \mathcal{F}_{(t+1 / n)+}$ such that its symmetric difference with $A$ is in $\mathcal{N}$. One deduces that the symmetric difference between $A$ and

$$
A^{\prime}:=\bigcap_{m \geq 1} \bigcup_{n \geq m} A_{n}^{\prime}
$$

is in $\mathcal{N}$. Now since for all integers $m_{0} \geq 1$

$$
A^{\prime}=\bigcap_{m \geq m_{0}} \bigcup_{n \geq m} A_{n}^{\prime}
$$

$A^{\prime} \in \mathcal{F}_{\left(t+1 / m_{0}\right)+}$ for all $m_{0} \geq 1$, which implies that $A^{\prime} \in \mathcal{F}_{t+}$, and then $A \in \widetilde{\mathcal{F}}_{t}$. We have thus proved that $\left(\Omega, \widetilde{\mathcal{F}},\left(\widetilde{\mathcal{F}}_{t}\right)_{t \geq 0}, \widetilde{\mathbb{P}}\right)$ satisfies the natural conditions; it remains to show that it is the smallest extension of $\left(\Omega, \mathcal{F},\left(\mathcal{F}_{t}\right)_{t>0}, \mathbb{P}\right)$ which enjoys this property. Let $\left(\Omega, \mathcal{F}^{\prime},\left(\mathcal{F}_{t}^{\prime}\right)_{t>0}, \mathbb{P}^{\prime}\right)$ be such an extension. By $\sigma$-completeness $\mathcal{F}_{0}^{\prime}$ contains all the $\sigma$-negligible sets of $\left(\Omega, \mathcal{F}^{\prime},\left(\mathcal{F}_{t}^{\prime}\right)_{t \geq 0}, \mathbb{P}^{\prime}\right)$ and a fortiori the $\sigma$-negligible sets of $\left(\Omega, \mathcal{F},\left(\mathcal{F}_{t}\right)_{t \geq 0}, \mathbb{P}\right)$. Moreover, for all $t \geq 0, \mathcal{F}_{t}^{\prime}=\mathcal{F}_{t+}^{\prime}$ by right-continuity, which implies that $\mathcal{F}_{t}^{\prime}$ contains $\mathcal{F}_{t+}$. Since it contains $\mathcal{N}$, it also contains $\widetilde{\mathcal{F}}_{t}$, and similarly, $\mathcal{F}^{\prime}$ contains $\widetilde{\mathcal{F}}$. Now, since $\mathbb{P}^{\prime}$ is an extension of $\mathbb{P}$, its restriction to $\widetilde{\mathcal{F}}$ is also an extension of $\mathbb{P}$ and by uniqueness it is necessarily equal to $\widetilde{\mathbb{P}}$. Hence, $\mathbb{P}^{\prime}$ is an extension of $\widetilde{\mathbb{P}}$.

Once the natural conditions are defined, it is natural to compare them with the usual conditions. One has the following result:

Proposition 2.5. Let $\left(\Omega, \mathcal{F},\left(\mathcal{F}_{t}\right)_{t \geq 0}, \mathbb{P}\right)$ be a filtered probability space which satisfies the natural conditions. Then for all $t \geq 0$, the space $\left(\Omega, \mathcal{F}_{t},\left(\mathcal{F}_{s}\right)_{0 \leq s \leq t}, \mathbb{P}\right)$ satisfies the usual conditions. 
Proof. The right-continuity of $\left(\mathcal{F}_{s}\right)_{0 \leq s \leq t}$ is obvious, let us prove the completeness. If $A$ is a negligible set of $\left(\Omega, \mathcal{F}_{t}, \mathbb{P}\right)$, there exists $B \in \mathcal{F}_{t}$, such that $A \subset B$ and $\mathbb{P}[B]=0$. One deduces immediately that $A$ is $\sigma$-negligible with respect to $\left(\Omega, \mathcal{F},\left(\mathcal{F}_{t}\right)_{t \geq 0}, \mathbb{P}\right)$, and by $\sigma$-completeness of this filtered probability space, $A \in \mathcal{F}_{0}$.

This relation between usual conditions and natural conditions is the main ingredient of the results in Section 3 where we prove that one can replace the usual conditions by the natural conditions in most of the classical results in stochastic calculus.

\section{Classical theorems under the natural CONDitions}

In the introduction we observed that it is very useful to have càdlàg versions of martingales. These versions always exist under the natural conditions:

Proposition 3.1. Let $\left(X_{t}\right)_{t \geq 0}$ be a submartingale or a supermartingale, with respect to a filtered probability space satisfying the natural conditions. If $\mathbb{E}\left[X_{t}\right]$ is right-continuous with respect to $t$ (in particular if $\left(X_{t}\right)_{t \geq 0}$ is a martingale), then $\left(X_{t}\right)_{t \geq 0}$ admits a càdlàg modification, which is unique up to indistinguishability.

Proof. Let us assume that $\left(X_{t}\right)_{t \geq 0}$ is defined on the filtered probability space $\left(\Omega, \mathcal{F},\left(\mathcal{F}_{t}\right)_{t \geq 0}, \mathbb{P}\right)$. For all $t \geq 0$, the process $\left(X_{s}\right)_{0 \leq s \leq t}$ is a submartingale or a supermartingale with respect to $\left(\Omega, \mathcal{F}_{t},\left(\mathcal{F}_{s}\right)_{0 \leq s \leq t}, \mathbb{P}\right)$ which satisfies the usual conditions since $\left(\Omega, \mathcal{F},\left(\mathcal{F}_{t}\right)_{t \geq 0}, \mathbb{P}\right)$ satisfies the natural conditions. By right-continuity of $\mathbb{E}\left[X_{t}\right]$ with respect to $t,\left(X_{s}\right)_{0 \leq s \leq t}$ admits a càdlàg modification $\left(\tilde{X}_{s}^{(t)}\right)_{0 \leq s \leq t}$, which is unique up to indistinguishability. This uniqueness implies that for $0 \leq t \leq u$, one has almost surely

$$
\tilde{X}_{s}^{(t)}=\tilde{X}_{s}^{(u)}
$$

for all $s \leq t$. Let us denote by $N$ the set of $\omega \in \Omega$ such that there exists integers $n \geq m \geq 1$, and $s \in[0, m]$, such that

$$
\tilde{X}_{s}^{(n)}(\omega) \neq \tilde{X}_{s}^{(m)}(\omega)
$$

It is easy to check that $N$ is $\sigma$-negligible, and then $N \in \mathcal{F}_{0}$ with $\mathbb{P}[N]=0$. One can now define a process $\left(\tilde{X}_{s}\right)_{s \geq 1}$ by

$$
\tilde{X}_{s}=\tilde{X}_{s}^{(n)}
$$

for $n \geq s \geq 0$, on the complement of $N$, and by $\tilde{X}_{s}=0$ for all $s \geq 0$, on $N$. This process is càdlàg, adapted (recall that $\left.N \in \mathcal{F}_{0}\right)$ and is a modification of $\left(X_{s}\right)_{s \geq 0}$. The uniqueness of this càdlàg modification is immediate.

There are many situations where one can construct càdlàg, or even continuous, versions of stochastic processes, but in general one cannot make sure that these versions are adapted to the natural filtration of the initial process. This problem can be solved by introducing the natural augmentation. Before stating the corresponding proposition let us prove the following useful lemma:

Lemma 3.2. Let $A>0$, and let $f$ be a real valued function defined on a dense subset $D$ of $[0, A]$, containing $A$. Then $f$ can be extended to a càdlàg function from $[0, A]$ to $\mathbb{R}$ if and only if the following two conditions hold:

- for all $x \in D \cap[0, A), f(y)$ tends to $f(x)$ for $y \in D$ going to $x$ from above;

- for all $\epsilon>0$, there exists $\delta>0$ such that for all $x, y, z \in D, x \leq y \leq z \leq x+\delta$ implies $|f(y)-f(x)| \wedge$ $|f(y)-f(z)| \leq \epsilon$.

If these conditions hold, the càdlàg extension of $f$ is unique. 
Proof. If the first condition given above is not satisfied, it is obvious that $f$ cannot be extended to a rightcontinuous function. Let us now suppose that the second condition does not hold. There exist $\epsilon>0$ and three sequences $\left(x_{n}\right)_{n \geq 0},\left(y_{n}\right)_{n \geq 0},\left(z_{n}\right)_{n \geq 0}$ such that $x_{n} \leq y_{n} \leq z_{n}$ for all $n \geq 0, z_{n}-x_{n}$ tends to zero when $n$ goes to infinity, and $\left|f\left(x_{n}\right)-f\left(y_{n}\right)\right| \wedge\left|f\left(y_{n}\right)-f\left(z_{n}\right)\right|>\epsilon$ for all $n \geq 0$. By taking subsequences, one can assume that $\left(y_{n}\right)_{n \geq 0}$ is monotone, and then converges to a limit $a \in[0, A]$. One has three possible cases:

- if $\left(y_{n}\right)_{n \geq 0}$ is non-decreasing, and $y_{n}<a$ for all $n \geq 0$, then $x_{n}<a$. Since $0 \leq y_{n}-x_{n} \leq z_{n}-x_{n}$ tends to zero when $n$ goes to infinity, $x_{n}$ tends to $a$, as $y_{n}$. We have proved that the two sequences $\left(x_{n}\right)_{n \geq 0}$ and $\left(y_{n}\right)_{n \geq 0}$ tend to $a$, strictly from below, but since $\left|f\left(x_{n}\right)-f\left(y_{n}\right)\right|>\epsilon$ for all $n, f$ cannot have a left limit at $a$;

- if $\left(y_{n}\right)_{n \geq 0}$ is non-decreasing, and $y_{n_{0}}=a$ for some integer $n_{0}$, then $y_{n}=a$ for all $n \geq n_{0}$, which implies $z_{n} \geq a$. Since $0 \leq z_{n}-y_{n} \leq z_{n}-x_{n}$ tends to zero when $n$ goes to infinity, $z_{n}$ tends to $a$. Hence $\left(z_{n}\right)_{n \geq 0}$ tend to $a$ from above, and since $\left|f\left(z_{n}\right)-f\left(y_{n}\right)\right|>\epsilon$ for all $n,\left|f\left(z_{n}\right)-f(a)\right|>\epsilon$ for all $n \geq n_{0}$, and $f(a)$ cannot be the right limit of $f$ at $a$;

- if $\left(y_{n}\right)_{n \geq 0}$ is non-increasing, $z_{n} \geq y_{n} \geq a$ for all $n \geq 0$, and $z_{n}$ tends to $a$ when $n$ goes to infinity. Hence, $\left(y_{n}\right)_{n \geq 0}$ and $\left(z_{n}\right)_{n \geq 0}$ tend to $a$ from above. Since $\left|f\left(z_{n}\right)-f\left(y_{n}\right)\right|>\epsilon$ for all $n$, $f$ has no right limit at $a$ if $a \notin D$, and $f(\bar{a})$ cannot be the right limit of $f$ at $a$ if $a \in D$.

In any case $f$ cannot be extended to a càdlàg function. On the other hand, let us suppose that $f$ has not a right limit at $a \in[0, A)$. Then, one can find a sequence $\left(t_{n}\right)_{n>0}$, strictly decreasing to $a$, such that one of these three properties holds:

- $f\left(t_{n+1}\right) \geq f\left(t_{n}\right)+1$ for all $n \geq 0$;

- $f\left(t_{n+1}\right) \leq f\left(t_{n}\right)-1$ for all $n \geq 0$;

- there exist $u, v \in \mathbb{R}$ such that for all $n \geq 0, f\left(t_{2 n}\right)<u<v<f\left(t_{2 n+1}\right)$.

In any case, $t_{2 n+2} \leq t_{2 n+1} \leq t_{2 n}$ for all $n \geq 0, t_{2 n}-t_{2 n+2}$ tends to zero when $n$ goes to infinity, but $\left|f\left(t_{2 n}\right)-f\left(t_{2 n+1}\right)\right| \wedge\left|f\left(t_{2 n+1}\right)-f\left(t_{2 n+2}\right)\right| \geq \epsilon$ for some $\epsilon>0$, independent of $n$. Hence the second condition given in Lemma 3.2 is not satisfied. One has a similar results if $f$ has no left limit at $a \in(0, A]$. Now let us assume that $f$ satisfies the two conditions given in Lemma 3.2. Necessarily, $f$ admits left and right limits everywhere. Let $g$ be the function from $[0, A]$ to $\mathbb{R}$ such that:

- for $t<A, g(t)$ is the right limit of $f$ at $t$;

- for $t=A, g(t)=f(A)$.

By assumption, for $t \in D \cap[0, A)$, the right limit of $f$ at $t$ is $f(t)$, and then $g$ coincides with $f$ on $D$. It remains to prove that $g$ is càdlàg. Let $t \in[0, A)$. For all $\epsilon>0$, there exists $u>t$ such that $|f(v)-g(t)| \leq \epsilon$ for all $v \in D \cap(t, u)$. Now for all $w \in[t, u), g(w)$ is the limit of $f(v)$ for $v \in D \cap(t, u)$ strictly decreasing to $w$, which implies that $|g(w)-g(t)| \leq \epsilon$. Hence, $g$ is right-continuous. Now, let $t \in(0, A]$, and let $b$ be the left limit of $f$ at $t$. For all $\epsilon>0$, there exists $u<t$ such that $|f(v)-b| \leq \epsilon$ for all $v \in D \cap(u, t)$. For $w \in(u, t), g(w)$ is the limit of $f(v)$ for $v \in D \cap(u, t)$ strictly decreasing to $w$, and then $|g(w)-b| \leq \epsilon$. Therefore, $g$ admits left limits. The uniqueness of the càdlàg extension of $f$ is due to the fact that two càdlàg functions from $[0, A]$ to $\mathbb{R}$ which coincide on a dense subset of $[0, A]$ containing $A$ are necessarily equal.

We are now able to prove the following result:

Proposition 3.3. Let $\left(\Omega, \mathcal{F},\left(\mathcal{F}_{t}\right)_{t \geq 0}, \mathbb{P}\right)$ be a filtered probability space satisfying the natural conditions, and let $\left(X_{t}\right)_{t \geq 0}$ be an adapted process defined on this space. We assume that there exists a càdlàg version $\left(Y_{t}\right)_{t \geq 0}$ of $\left(X_{t}\right)_{t \geq 0}$. Then there exists a càdlàg and adapted version of $\left(X_{t}\right)_{t \geq 0}$, which is necessarily indistinguishable from $\left(Y_{t}\right)_{t \geq 0}$.

Proof. Let $D$ be a countable and dense subset of $\mathbb{R}_{+}$, containing $\mathbb{N}$, and for all integers $n \geq 1$, let $N_{n}$ be the set of $\omega \in \Omega$ such that the function $f$ from $D \cap[0, n]$ to $\mathbb{R}$, defined by $f(t)=X_{t}(\omega)$, does not admit a unique càdlàg extension to $[0, n]$. By Lemma $3.2, N_{n} \in \mathcal{F}_{n}$ for all $n \geq 0$, since $\left(X_{t}\right)_{t \geq 0}$ is adapted. Now, $\left(Y_{t}\right)_{t \geq 0}$ is càdlàg, which implies that for all $n$

$$
N_{n} \subset\left\{\exists t \in D, X_{t} \neq Y_{t}\right\} .
$$


Since $\left(Y_{t}\right)_{t \geq 0}$ is a version of $\left(X_{t}\right)_{t \geq 0}$, one deduces that $\mathbb{P}\left[N_{n}\right]=0$ for all $n$. Hence

$$
N:=\bigcup_{n \geq 0} N_{n}
$$

is $\sigma$-negligible, which implies that $N \in \mathcal{F}_{0}$ and $\mathbb{P}[N]=0$. Now, let $\omega \notin N$, and for $n \geq 1$, let $g_{\omega, n}$ be the unique càdlàg extension to $[0, n]$ of the function $f_{\omega, n}$ from $D \cap[0, n]$ to $\mathbb{R}$, defined by $f_{\omega, n}(t)=X_{t}(\omega)$. By uniqueness, $g_{\omega, m}$ and $g_{\omega, n}$ coincide on $[0, m]$ for $m \leq n$. Hence there exists a càdlàg function $g_{\omega}$ from $\mathbb{R}_{+}$to $\mathbb{R}$ such that $g_{\omega}(t)=X_{t}(\omega)$ for all $t \in D$. Now, let $\left(\tilde{X}_{t}\right)_{t \geq 0}$ be the process defined by:

$$
\tilde{X}_{t}(\omega)=g_{\omega}(t) \mathbb{1}_{\omega \notin N},
$$

which is càdlàg (on $N$ it is identically zero). For all $t \geq 0$, and for any sequence $\left(t_{n}\right)_{n \geq 0}$ of elements of $D$, tending to $t$ from above

$$
\tilde{X}_{t}(\omega)=\mathbb{1}_{\omega \notin N} \lim _{n \rightarrow \infty} g_{\omega}\left(t_{n}\right)=\mathbb{1}_{\omega \notin N} \lim _{n \rightarrow \infty} X_{t_{n}}(\omega) .
$$

Since $\left(X_{t}\right)_{t \geq 0}$ is adapted, $\left(\mathcal{F}_{t}\right)_{t \geq 0}$ is right-continuous and $N \in \mathcal{F}_{0},\left(\tilde{X}_{t}\right)_{t \geq 0}$ is adapted. Moreover for all $t \geq 0$, $\tilde{X}_{t}$ is almost surely the right limit of $Y$ at $t$, restricted to $D$, since $Y$ is a version of $X$ and $N$ is negligible. Since $Y$ is càdlàg, $\tilde{X}_{t}=Y_{t}$ a.s., and $\tilde{X}_{t}=X_{t}$ almost surely. Consequently $\tilde{X}$ is a càdlàg and adapted version of $X$. Now two càdlàg versions of $X$ are necessarily indistinguishable, since there almost surely coincide at all $t \in D$.

One has a similar result for continuous processes:

Proposition 3.4. Let $\left(\Omega, \mathcal{F},\left(\mathcal{F}_{t}\right)_{t \geq 0}, \mathbb{P}\right)$ be a filtered probability space satisfying the natural conditions, and let $\left(X_{t}\right)_{t \geq 0}$ be an adapted process defined on this space. We assume that there exists a continuous version $\left(Y_{t}\right)_{t \geq 0}$ of $\left(X_{t}\right)_{t \geq 0}$. Then there exists a continuous and adapted version of $\left(X_{t}\right)_{t \geq 0}$, which is necessarily indistinguishable from $\left(Y_{t}\right)_{t \geq 0}$.

Proof. It is similar to the proof of Proposition 3.3, and more simple, so we go quickly. If $D$ is a countable and dense subset of $\mathbb{R}_{+}$, the restriction of $X$ to $D$ is, except on an $\sigma$-negligible set $N$, locally, uniformly continuous, since $X$ has a continuous version. Therefore one can define a continuous, adapted version of $X$ by taking its limit (after restriction to $D$ ), on the complement of $N$, and zero on $N$.

Similarly one has the following version of the Kolmogorov criteria:

Proposition 3.5. Let $\left(\Omega, \mathcal{F},\left(\mathcal{F}_{t}\right)_{t \geq 0}, \mathbb{P}\right)$ be a filtered probability space satisfying the natural conditions, and let $\left(X_{t}\right)_{t \geq 0}$ be an adapted process defined on this space. We suppose that there exist $\alpha, \beta>0$, and for all $A>0$, $C(A)>0$, such that for $0 \leq s \leq t \leq A$ :

$$
\mathbb{E}_{\mathbb{P}}\left[\left|X_{t}-X_{s}\right|^{\alpha}\right] \leq C(A)(t-s)^{1+\beta} .
$$

Then $\left(X_{t}\right)_{t \geq 0}$ admits an adapted version which is locally Hölder of any index strictly smaller than $\beta / \alpha$. This version is unique up to indistinguishability.

Proof. Let $D$ be a countable, dense subset of $\mathbb{R}_{+}$. By the classical Kolmogorov criteria, there exists a version $Y$ of $X$ which is locally Hölder of any index strictly smaller than $\beta / \alpha$. One deduces that except on an $\sigma$-negligible set, the restriction of $X$ to $D$ is also locally Hölder of any index strictly smaller than $\beta / \alpha$. One then extends this restriction to all $\mathbb{R}_{+}$by continuity, which gives an adapted and Hölder version of $X$. 
One also has a version of the début theorem:

Proposition 3.6. Let $\left(\Omega, \mathcal{F},\left(\mathcal{F}_{t}\right)_{t \geq 0}, \mathbb{P}\right)$ be a filtered probability space satisfying the natural conditions, and let $A$ be a progressive subset of $\mathbb{R}_{+} \times \Omega$. Then the début of $A$, i.e. the random time $D(A)$ such that for all $\omega \in \Omega$ :

$$
D(A)(\omega):=\inf \{t \geq 0,(t, \omega) \in A\}
$$

is an $\left(\mathcal{F}_{t}\right)_{t \geq 0}$-stopping time.

Proof. Let $t \geq 0$. The set

$$
A^{(t)}:=A \cap([0, t] \times \Omega)
$$

is a progressive set of $[0, t] \times \Omega$, with respect to the filtered probability space $\left(\Omega, \mathcal{F}_{t},\left(\mathcal{F}_{s}\right)_{0 \leq s \leq t}, \mathbb{P}\right)$. Since this space satisfies the usual conditions, one can apply the classical début theorem, which implies that the début $D\left(A^{(t)}\right)$ of $A^{(t)}$ is a stopping time. Now, one immediately checks that $D(A)<t$ if and only if $D\left(A^{(t)}\right)<t$ which is in $\mathcal{F}_{t}$. Since $\left(\mathcal{F}_{t}\right)_{t \geq 0}$ is right-continuous, $D(A)$ is a stopping time.

One has the following corollary:

Corollary 3.7. Let $\left(X_{t}\right)_{t \geq 0}$ be a progressively measurable process defined on a filtered probability space which satisfies the natural conditions (the condition of progressive measurability is satisfied, in particular, if $\left(X_{t}\right)_{t \geq 0}$ is adapted and càdlàg). Then, for all Borel sets $A \subset \mathbb{R}$ :

$$
T_{A}:=\inf \left\{t \geq 0, X_{t} \in A\right\}
$$

is an $\left(\mathcal{F}_{t}\right)_{t \geq 0}$-stopping time.

Proof. The random time $T_{A}$ is the début of the set:

$$
\left\{(t, \omega) \in \mathbb{R}_{+} \times \Omega, X_{t}(\omega) \in A\right\},
$$

which is progressive, since $\left(X_{t}\right)_{t \geq 0}$ is progressively measurable.

Under the natural conditions, one can also prove the existence of the Doob-Meyer decomposition for submartingales. In particular this implies the existence of a càdlàg (in fact continuous) and adapted version of the Brownian local time at level zero.

Proposition 3.8. Let $\left(X_{t}\right)_{t \geq 0}$ be a right-continuous submartingale defined on a filtered probability space $(\Omega, \mathcal{F}$, $\left.\left(\mathcal{F}_{t}\right)_{t \geq 0}, \mathbb{P}\right)$, satisfying the natural conditions. We suppose that $\left(X_{t}\right)_{t \geq 0}$ is of class $(D L)$, i.e. for all a $\geq 0$, $\left(X_{T}\right)_{T \in \mathcal{T}_{a}}$ is uniformly integrable, where $\mathcal{T}_{a}$ is the family of the $\left(\mathcal{F}_{t}\right)_{t \geq 0}$-stopping times which are bounded by a (for example, every nonnegative submartingale is of class $(D L)$ ). Then, there exists a right-continuous $\left(\mathcal{F}_{t}\right)_{t \geq 0}-$ martingale $\left(M_{t}\right)_{t \geq 0}$ and an increasing process $\left(A_{t}\right)_{t \geq 0}$ starting at zero, such that:

$$
X_{t}=M_{t}+A_{t}
$$

for all $t \geq 0$, and for every bounded, right-continuous martingale $\left(\xi_{s}\right)_{s \geq 0}$,

$$
\mathbb{E}\left[\xi_{t} A_{t}\right]=\mathbb{E}\left[\int_{(0, t]} \xi_{s-} \mathrm{d} A_{s}\right],
$$

where $\xi_{s-}$ is the left-limit of $\xi$ at $s$, almost surely well-defined for all $s>0$. The processes $\left(M_{t}\right)_{t \geq 0}$ and $\left(A_{t}\right)_{t \geq 0}$ are uniquely determined, up to indistinguishability. Moreover, they can be chosen to be continuous if $\left(X_{t}\right)_{t \geq 0}$ is a continuous process. 
Proof. Since for all $t \geq 0,\left(\Omega, \mathcal{F}_{t},\left(\mathcal{F}_{s}\right)_{0 \leq s \leq t}, \mathbb{P}\right)$ satisfies the usual conditions, there exists $\left(M_{s}^{(t)}\right)_{0 \leq s \leq t}$, a rightcontinuous $\left(\mathcal{F}_{s}\right)_{0 \leq s \leq t}$-martingale and $\left(A_{s}^{(t)}\right)_{0 \leq s \leq t}$ increasing, such that $X_{s}=M_{s}^{(t)}+A_{s}^{(t)}$ for all $s \leq t$, and for all $u \in[0, t]$, and all bounded, right-continuous martingales $\left(\xi_{s}\right)_{s \geq 0}$ :

$$
\mathbb{E}\left[\xi_{u} A_{u}^{(t)}\right]=\mathbb{E}\left[\int_{(0, u]} \xi_{s-} \mathrm{d} A_{s}^{(t)}\right] .
$$

Moreover, one can suppose that $\left(M_{s}^{(t)}\right)_{0 \leq s \leq t}$ and $\left(A_{s}^{(t)}\right)_{0 \leq s \leq t}$ are continuous if $\left(X_{s}\right)_{s \geq 0}$ is continuous. By uniqueness of $\left(M_{s}^{(t)}\right)_{0 \leq s \leq t}$ there exists an $\sigma$-negligible set $N$ such that for all $\omega \notin N$, for all integers $n \geq m \geq 1$, and for all $s \in[0, m]$ :

$$
M_{s}^{(n)}(\omega)=M_{s}^{(m)}(\omega)
$$

and

$$
A_{s}^{(n)}(\omega)=A_{s}^{(m)}(\omega)
$$

Since $N$ is $\sigma$-negligible, $N \in \mathcal{F}_{0}$ with $\mathbb{P}[N]=0$. One can now define the processes $\left(M_{s}\right)_{s \geq 0}$ and $\left(A_{s}\right)_{s \geq 0}$ by $M_{s}=M_{s}^{(n)}, A_{s}=A_{s}^{(n)}$ for $n \geq s \geq 0$, on the complement of $N$, and by $M_{s}=X_{s}, A_{s}=0$ for all $s \geq 0$, on $N$. In particular, $\left(M_{s}\right)_{s \geq 0}$ and $\left(A_{s}\right)_{s \geq 0}$ are continuous if $\left(X_{s}\right)_{s \geq 0}$ is continuous.

One deduces the following corollary (giving quadratic variation):

Corollary 3.9. Let $\left(M_{t}\right)_{t \geq 0}$ be a continuous, square-integrable martingale defined on a filtered probability space $\left(\Omega, \mathcal{F},\left(\mathcal{F}_{t}\right)_{t \geq 0}, \mathbb{P}\right)$, satisfying the natural conditions. Then, there exists a unique continuous, increasing process $\left(\langle M\rangle_{t}\right)_{t \geq 0}$, starting from zero, such that $\left(M_{t}^{2}-\langle M\rangle_{t}\right)_{t \geq 0}$ is a martingale.

The quadratic variation is involved in a very important way in the construction of the stochastic integral, which can also be made under natural conditions. We do not give here the details of the different constructions of the stochastic integral, but the general way to go from the usual to the natural conditions is the following: let us suppose that under the usual conditions, one can construct a stochastic integral of the form:

$$
\left(\int_{0}^{s} H_{u} \mathrm{~d} X_{u}\right)_{s \geq 0}
$$

as a càdlàg, adapted process. Then, under the natural conditions, one can also define

$$
\left(\int_{0}^{s} H_{u} \mathrm{~d} X_{u}\right)_{0 \leq s \leq t}
$$

as a càdlàg, adapted process $\left(I_{s}^{(t)}\right)_{0 \leq s \leq t}$, since the restriction to $[0, t]$ of the underlying filtration satisfies the usual conditions. Now, let us assume that for $t^{\prime}>t>0$, the restriction of $\left(I_{s}^{\left(t^{\prime}\right)}\right)_{0 \leq s \leq t^{\prime}}$ to the interval $[0, t]$ is indistinguishable from the process $\left(I_{s}^{(t)}\right)_{0 \leq s \leq t}$. Since the processes are càdlàg, this assumption is implied by the fact that for all $s, t, t^{\prime}$ such that $t^{\prime} \geq t \geq s \geq 0, I_{s}^{(t)}=I_{s}^{\left(t^{\prime}\right)}$ almost surely, which is reasonable since $I_{s}^{(t)}$ and $I_{s}^{\left(t^{\prime}\right)}$ correspond to two constructions of the same stochastic integral:

$$
\int_{0}^{s} H_{u} \mathrm{~d} X_{u}
$$

If this almost sure equality can be proved rigorously (this can be checked in each specific construction of the stochastic integral), one deduces that except on a $\sigma$-negligible set $N$, all the processes $\left(I_{s}^{(n)}\right)_{0 \leq s \leq n}$, for $n \in \mathbb{N}$, are 
restrictions of each other. By defining, for all $s \geq 0, I_{s}$ as zero on $N$, and as $I_{s}^{(n)}$ for $n \geq s$ on the complement of $N$, one obtains a càdlàg, adapted process, such that for $t \geq s \geq 0$ :

$$
I_{s}=I_{s}^{(t)}
$$

almost surely, which gives a construction of the stochastic integral under the natural conditions.

Two other important results are the section theorem and the projection theorem. These results are proved in [3] if the probability space is complete. In fact, they remain true if the space is only supposed to be $\sigma$-complete. More precisely, the section theorem can be stated as follows:

Proposition 3.10. Let $\left(\Omega, \mathcal{F},\left(\mathcal{F}_{t}\right)_{t>0}, \mathbb{P}\right)$ be a $\sigma$-complete filtered probability space. Let $A$ be a optional subset of $\mathbb{R}_{+} \times \Omega$. Then the image $\pi(A)$ of $A$ by the projection from $\mathbb{R}_{+} \times \Omega$ to $\Omega$ is in $\mathcal{F}$, and for all $\epsilon>0$, there exists a stopping time $T$ enjoying the following two properties:

- for all $\omega \in \Omega$ such that $T(\omega)<\infty,(T(\omega), \omega) \in A$;

- $\mathbb{P}[T<\infty] \geq \mathbb{P}[\pi(A)]-\epsilon$.

Proof. Let $\epsilon>0$ and $u \in \mathbb{R}_{+}$. The negligible sets of $\left(\Omega, \mathcal{F}_{u}, \mathbb{P}\right)$ are $\sigma$-negligible with respect to $\left(\Omega, \mathcal{F},\left(\mathcal{F}_{t}\right)_{t>0}, \mathbb{P}\right)$, hence, they are in $\mathcal{F}_{0}$ : the probability space $\left(\Omega, \mathcal{F}_{u}, \mathbb{P}\right)$ is complete. Moreover, the set $A \cap([0, u) \times \Omega)$ is optional with respect to $\left(\Omega, \mathcal{F}_{u},\left(\mathcal{F}_{t \wedge u}\right)_{t \geq 0}, \mathbb{P}\right)$. This is a consequence of the following two facts:

- the family of sets $B \subset \mathbb{R}_{+} \times \Omega$ such that $B \cap([0, u) \times \Omega)$ is optional with respect to $\left(\Omega, \mathcal{F}_{u},\left(\mathcal{F}_{t \wedge u}\right)_{t \geq 0}, \mathbb{P}\right)$ is a $\sigma$-algebra;

- for all stopping times $T$ with respect to $\left(\Omega, \mathcal{F},\left(\mathcal{F}_{t}\right)_{t \geq 0}, \mathbb{P}\right)$, the set of $(t, \omega) \in[0, u) \times \Omega$ such that $T(\omega) \leq t$ is optional with respect to $\left(\Omega, \mathcal{F}_{u},\left(\mathcal{F}_{t \wedge u}\right)_{t \geq 0}, \mathbb{P}\right)$.

The first property is proved as follows:

- if $B_{n} \cap([0, u) \times \Omega)$ is optional for all integers $n \geq 1$ :

$$
\left(\bigcup_{n \geq 1} B_{n}\right) \cap([0, u) \times \Omega)=\bigcup_{n \geq 1}\left[B_{n} \cap([0, u) \times \Omega)\right]
$$

is optional;

- if $B \cap([0, u) \times \Omega)$ is optional, and if $C$ is the complement of $B$, then $C \cap([0, u) \times \Omega)$ is the intersection of $[0, u) \times \Omega$ and the complement of $B \cap([0, u) \times \Omega)$, hence, it is optional.

The set involved in the second property corresponds to the stochastic interval $[T, u)$ for $T<u$, and the empty set for $T \geq u$. Equivalently it corresponds to the intersection of $[T \wedge u, \infty)$ and $[0, u)$, and then is optional since $T \wedge u$ is a stopping time with respect to the filtration $\left(\mathcal{F}_{t \wedge u}\right)_{t \geq 0}$. We have now proved that for all integers $n \geq 1, A_{n}:=A \cap([0, n) \times \Omega)$ is optional with respect to $\left(\Omega, \mathcal{F}_{n},\left(\mathcal{F}_{t \wedge n}\right)_{t \geq 0}, \mathbb{P}\right)$. By the section theorem in [3], $A_{n}$ is measurable and there exists an $\left(\mathcal{F}_{t \wedge n}\right)_{t \geq 0}$-stopping time $T_{n}$ such that $T_{n}(\omega)<\infty \operatorname{implies}\left(T_{n}(\omega), \omega\right) \in A_{n}$ and $\mathbb{P}\left[T_{n}<\infty\right] \geq \mathbb{P}\left[\pi\left(A_{n}\right)\right]-\left(\epsilon / 2^{n}\right)$, or equivalently,

$$
\mathbb{P}\left[\pi\left(A_{n}\right) \backslash\left\{T_{n}<\infty\right\}\right] \leq \epsilon / 2^{n},
$$

since $\pi\left(A_{n}\right)$ contains the event $\left\{T_{n}<\infty\right\}$. Now $\pi(A)$ is the increasing union of $\pi\left(A_{n}\right)$ for $n \geq 1$, hence, it is in $\mathcal{F}$. Moreover there exists $N \geq 1$ such that $\mathbb{P}\left[\pi\left(A_{N}\right)\right] \geq \mathbb{P}[\pi(A)]-\epsilon$. Let $T$ be the infimum of $T_{n}$ for $1 \leq n \leq N$ : it is an $\left(\mathcal{F}_{t}\right)_{t \geq 0}$-stopping time. If $T$ is finite, let $n$ be an index such that $T=T_{n}$; one has $\left(T_{n}(\omega), \omega\right) \in A_{n}$, 
which implies that $(T(\omega), \omega) \in A$. Moreover

$$
\begin{aligned}
\mathbb{P}\left[\pi\left(A_{N}\right) \backslash\{T<\infty\}\right] & =\mathbb{P}\left[\left(\bigcup_{1 \leq n \leq N} \pi\left(A_{n}\right)\right) \cap\left(\bigcap_{1 \leq n \leq N}\left\{T_{n}=\infty\right\}\right)\right] \\
& \leq \sum_{1 \leq n \leq N} \mathbb{P}\left[\pi\left(A_{n}\right) \cap\left\{T_{n}=\infty\right\}\right] \\
& \leq \epsilon
\end{aligned}
$$

Therefore

$$
\mathbb{P}[T<\infty] \geq \mathbb{P}\left[\pi\left(A_{N}\right)\right]-\epsilon \geq \mathbb{P}[A]-2 \epsilon .
$$

We deduce from the section theorem the following version of the projection theorem:

Proposition 3.11. Let $\left(X_{t}\right)_{t \geq 0}$ be a bounded, measurable process, defined on a $\sigma$-complete, filtered probability space $\left(\Omega, \mathcal{F},\left(\mathcal{F}_{t}\right)_{t \geq 0}, \mathbb{P}\right)$. Then, there exists an optional stochastic process $\left(Y_{t}\right)_{t \geq 0}$ such that for all stopping times $T$ :

$$
\mathbb{E}\left[X_{T} \mathbb{1}_{T<\infty} \mid \mathcal{F}_{T}\right]=Y_{T} \mathbb{1}_{T<\infty} .
$$

This process is unique up to indistinguishability.

Proof. The proof in [3] is sufficient here, since it uses completeness of the space only via the section theorem.

\section{EXtension of MeAsures AND PRoperties PRESERVED By the NATURAL AUGMENTATION}

A natural question which arises when one has, on a filtered probability space $\left(\Omega, \mathcal{F},\left(\mathcal{F}_{t}\right)_{t \geq 0}, \mathbb{P}\right)$, a coherent family $\left(\mathbb{Q}_{t}\right)_{t \geq 0}$ of probability measures, $\mathbb{Q}_{t}$ defined on $\mathcal{F}_{t}$, is the following: does there exist a probability measure $\mathbb{Q}$ defined on $\mathcal{F}$, such that its restriction to $\mathcal{F}_{t}$ is $\mathbb{Q}_{t}$ for all $t \geq 0$ ? As we have seen in the introduction, the answer to this question is not obvious at all and can be negative in very simple cases. However, one can state sufficient conditions on the space $\left(\Omega, \mathcal{F},\left(\mathcal{F}_{t}\right)_{t \geq 0}, \mathbb{P}\right)$, under which the answer is always positive. This problem is not new and has already received attention in the literature: it is dealt with in great detail by Parthasarathy in [9]. In particular the conditions we are going to state are not new and are already contained in [9] (p. 141). We nevertheless provide a proof, first because our method is slightly different and uses the existence of a regular conditional probability measure to construct the measure on $\mathcal{F}_{\infty}$, and second because this type of result does not seem to be well known. To be complete, one should add that Föllmer, in [6], has also studied a similar problem in a more general context and has obtained sufficient conditions relying on Parthasarathy's results. However the results there would not apply here: in particular the conditions in [6] are not satisfied by the space of continuous functions and the space of càdlàg functions, equipped with the natural filtration of the canonical process (these filtrations are not standard filtrations with the definition of a standard filtration given in [6]).

We first start with some definitions and then give sufficient conditions on a filtered measurable space $\left(\Omega, \mathcal{F},\left(\mathcal{F}_{t}\right)_{t \geq 0}\right)$ in order to have a positive answer to the extension of measure problem. Then we show that when this extension is possible, then it still holds under the natural augmentation with respect to some probability measure. Whenever we need a reference for probability measures on metric spaces, we cite [9] or the first chapter of $[10]$. 
Definition 4.1. Let $\left(\Omega, \mathcal{F},\left(\mathcal{F}_{t}\right)_{t \geq 0}\right)$ be a filtered measurable space, such that $\mathcal{F}$ is the $\sigma$-algebra generated by $\mathcal{F}_{t}, t \geq 0: \mathcal{F}=\bigvee_{t \geq 0} \mathcal{F}_{t}$. We shall say that the property $(\mathrm{P})$ holds if and only if $\left(\mathcal{F}_{t}\right)_{t \geq 0}$ enjoys the following conditions:

- for all $t \geq 0, \mathcal{F}_{t}$ is generated by a countable number of sets;

- for all $t \geq 0$, there exists a Polish space $\Omega_{t}$, and a surjective map $\pi_{t}$ from $\Omega$ to $\Omega_{t}$, such that $\mathcal{F}_{t}$ is the $\sigma$-algebra of the inverse images, by $\pi_{t}$, of Borel sets in $\Omega_{t}$, and such that for all $B \in \mathcal{F}_{t}, \omega \in \Omega$, $\pi_{t}(\omega) \in \pi_{t}(B)$ implies $\omega \in B$

- if $\left(\omega_{n}\right)_{n \geq 0}$ is a sequence of elements of $\Omega$, such that for all $N \geq 0$,

$$
\bigcap_{n=0}^{N} A_{n}\left(\omega_{n}\right) \neq \emptyset
$$

where $A_{n}\left(\omega_{n}\right)$ is the intersection of the sets in $\mathcal{F}_{n}$ containing $\omega_{n}$, then:

$$
\bigcap_{n=0}^{\infty} A_{n}\left(\omega_{n}\right) \neq \emptyset \text {. }
$$

Given this technical definition, one can state the following result:

Proposition 4.2. Let $\left(\Omega, \mathcal{F},\left(\mathcal{F}_{t}\right)_{t \geq 0}\right)$ be a filtered measurable space satisfying the property $(P)$, and let, for $t \geq 0, \mathbb{Q}_{t}$ be a probability measure on $\left(\Omega, \mathcal{F}_{t}\right)$, such that for all $t \geq s \geq 0, \mathbb{Q}_{s}$ is the restriction of $\mathbb{Q}_{t}$ to $\mathcal{F}_{s}$. Then, there exists a unique measure $\mathbb{Q}$ on $(\Omega, \mathcal{F})$ such that for all $t \geq 0$, its restriction to $\mathcal{F}_{t}$ is equal to $\mathbb{Q}_{t}$.

Remark 4.3. It is proven in $[8]$ (p. 166), Theorem 3.4, that if on the filtered measurable space $\left(\Omega, \mathcal{F},\left(\mathcal{F}_{t}\right)_{t \geq 0}\right)$, where $\left(\mathcal{F}_{t}\right)_{t>0}$ is right-continuous, we are given two probability measures $\mathbb{Q}$ and $\mathbb{Q}^{\prime}$ such that $\mathbb{Q}$ is locally absolutely continuous with respect to $\mathbb{Q}^{\prime}$, then there exists a $\mathbb{Q}^{\prime}$-martingale $M$ such that $M_{t}=\mathrm{d} \mathbb{Q}_{t} / \mathrm{d} \mathbb{Q}_{t}^{\prime}\left(\mathbb{Q}_{t}\right.$ and $\mathbb{Q}_{t}^{\prime}$ are the restrictions of the measures $\mathbb{Q}$ and $\mathbb{Q}^{\prime}$ to $\mathcal{F}_{t}$ ). The above proposition shows that this theorem admits a reciprocal if the filtered measurable space $\left(\Omega, \mathcal{F},\left(\mathcal{F}_{t}\right)_{t \geq 0}\right)$ satisfies property $(\mathrm{P})$.

Proof. The uniqueness is a direct application of the monotone class theorem. Let us prove the existence. For $t \geq 0$, the map $\pi_{t}$ is by assumption measurable from $\left(\Omega, \mathcal{F}_{t}\right)$ to $\left(\Omega_{t}, \mathcal{B}\left(\Omega_{t}\right)\right.$ ) (where $\mathcal{B}\left(\Omega_{t}\right)$ is the Borel $\sigma$-algebra of $\Omega_{t}$ ). Let $\overline{\mathbb{Q}}_{t}$ be the image of $\mathbb{Q}_{t}$ by $\pi_{t}$. By Theorem 1.1.6 of [10], for $0 \leq s \leq t$, there exists a conditional probability distribution of $\overline{\mathbb{Q}}_{t}$ given the $\sigma$-algebra $\pi_{t}\left(\mathcal{F}_{s}\right)$, generated by the images by $\pi_{t}$ of the sets in $\mathcal{F}_{s}$. Note that this $\sigma$-algebra is included in $\mathcal{B}\left(\Omega_{t}\right)$. Indeed, if $B \in \mathcal{F}_{s}$, there exists $A \in \mathcal{B}\left(\Omega_{t}\right)$ such that $B=\pi_{t}^{-1}(A)$, and then $\pi_{t}(B)=\pi_{t} \circ \pi_{t}^{-1}(A)$, which is equal to $A$ by the surjectivity of $\pi_{t}$. Now, the existence of the conditional probability distribution described above means that one can find a family $\left(Q_{\omega}\right)_{\omega \in \Omega_{t}}$ of probability measures on $\left(\Omega_{t}, \mathcal{B}\left(\Omega_{t}\right)\right)$ such that:

- for each $B \in \mathcal{B}\left(\Omega_{t}\right), \omega \rightarrow Q_{\omega}(B)$ is $\pi_{t}\left(\mathcal{F}_{s}\right)$-measurable;

- for every $A \in \pi_{t}\left(\mathcal{F}_{s}\right), B \in \mathcal{B}\left(\Omega_{t}\right)$ :

$$
\overline{\mathbb{Q}}_{t}(A \cap B)=\int_{A} Q_{\omega}(B) \overline{\mathbb{Q}}_{t}(\mathrm{~d} \omega) .
$$

Now, for all $\omega \in \Omega$, let us define the map $R_{\omega}$ from $\mathcal{F}_{t}$ to $\mathbb{R}_{+}$, by

$$
R_{\omega}[B]=Q_{\pi_{t}(\omega)}\left[\pi_{t}(B)\right]
$$

The map $R_{\omega}$ is a probability measure on $\left(\Omega, \mathcal{F}_{t}\right)$. Indeed,

$$
R_{\omega}[\Omega]=Q_{\pi_{t}(\omega)}\left[\Omega_{t}\right]=1,
$$


since $\pi_{t}$ is surjective. Moreover, let $\left(B_{k}\right)_{k \geq 1}$ be a family of disjoint sets in $\mathcal{F}_{t}$, and $B_{0}$ their union. By assumption, there exist $\left(\tilde{B}_{k}\right)_{k \geq 0}$ in $\mathcal{B}\left(\Omega_{t}\right)$ such that $B_{k}=\pi_{t}^{-1}\left(\tilde{B}_{k}\right)$. Since $\pi_{t}$ is surjective, $\pi_{t}\left(B_{k}\right)=\tilde{B}_{k}$. Moreover, the sets $\left(\tilde{B}_{k}\right)_{k \geq 1}$ are pairwise disjoint. Indeed, if $x \in \tilde{B}_{k} \cap \tilde{B}_{l}$ for $k>l \geq 1$, then, by surjectivity, there exists $y \in \Omega$, such that $x=\pi_{t}(y)$, which implies $y \in \pi_{t}^{-1}\left(\tilde{B}_{k}\right) \cap \pi_{t}^{-1}\left(\tilde{B}_{l}\right)$, and then $y \in B_{k} \cap B_{l}$, which is impossible. Therefore:

$$
\begin{aligned}
R_{\omega}\left[B_{0}\right] & =Q_{\pi_{t}(\omega)}\left[\tilde{B}_{0}\right] \\
& =\sum_{k \geq 1} Q_{\pi_{t}(\omega)}\left[\tilde{B}_{k}\right] \\
& =\sum_{k \geq 1} R_{\omega}\left[B_{k}\right] .
\end{aligned}
$$

Hence, $R_{\omega}$ is a probability measure. Moreover, for each $B \in \mathcal{F}_{t}$, the map $\omega \rightarrow R_{\omega}(B)$ is the composition of the measurable maps $\omega \rightarrow \pi_{t}(\omega)$ from $\left(\Omega, \mathcal{F}_{s}\right)$ to $\left(\Omega_{t}, \pi_{t}\left(\mathcal{F}_{s}\right)\right)$, and $\omega^{\prime} \rightarrow Q_{\omega^{\prime}}\left[\pi_{t}(B)\right]$ from $\left(\Omega_{t}, \pi_{t}\left(\mathcal{F}_{s}\right)\right)$ to $\left(\mathbb{R}_{+}, \mathcal{B}\left(\mathbb{R}_{+}\right)\right)$, and hence, it is $\mathcal{F}_{s}$-measurable. The measurability of $\pi_{t}$ follows from the fact that for $A \in \mathcal{F}_{s}$, the inverse image of $\pi_{t}(A)$ by $\pi_{t}$ is exactly $A$ (by an assumption given in the definition of the property $(\mathrm{P})$ ). Moreover, for every $A \in \mathcal{F}_{s}, B \in \mathcal{F}_{t}$ :

$$
\begin{aligned}
\mathbb{Q}_{t}(A \cap B) & =\mathbb{Q}_{t}\left[\left(\pi_{t}^{-1} \circ \pi_{t}(A)\right) \cap\left(\pi_{t}^{-1} \circ \pi_{t}(B)\right)\right] \\
& =\mathbb{Q}_{t}\left[\pi_{t}^{-1}\left(\pi_{t}(A) \cap \pi_{t}(B)\right)\right] \\
& =\overline{\mathbb{Q}}_{t}\left[\pi_{t}(A) \cap \pi_{t}(B)\right] \\
& =\int_{\Omega_{t}} \mathbb{1}_{\omega \in \pi_{t}(A)} Q_{\omega}\left(\pi_{t}(B)\right) \overline{\mathbb{Q}}_{t}(\mathrm{~d} \omega) \\
& =\int_{\Omega} \mathbb{1}_{\pi_{t}(\omega) \in \pi_{t}(A)} Q_{\pi_{t}(\omega)}\left(\pi_{t}(B)\right) \mathbb{Q}_{t}(\mathrm{~d} \omega) \\
& =\int_{A} R_{\omega}(B) \mathbb{Q}_{t}(\mathrm{~d} \omega) .
\end{aligned}
$$

Finally, we have found a conditional probability distribution of $\mathbb{Q}_{t}$ with respect to $\mathcal{F}_{s}$. Since $\mathcal{F}_{s}$ is countably generated, this conditional probability distribution is regular, by Theorem 1.1.8 in [10]. One can then apply Theorem 1.1.9, again in [10], and since $\mathcal{F}$ is the $\sigma$-algebra generated by $\mathcal{F}_{t}, t \geq 0$, one obtains a probability distribution $\mathbb{Q}$ on $(\Omega, \mathcal{F})$ such that for all integers $n \geq 0$, the restriction of $\mathbb{Q}$ to $\mathcal{F}_{n}$ is $\mathbb{Q}_{n}$. Now, for $t \geq 0$, let $\Lambda_{t}$ be an event in $\mathcal{F}_{t}$. One has, for $n>t$, integer:

$$
\mathbb{Q}\left[\Lambda_{t}\right]=\mathbb{Q}_{n}\left[\Lambda_{t}\right]=\mathbb{Q}_{t}\left[\Lambda_{t}\right]
$$

which implies that $\mathbb{Q}$ satisfies the assumptions of Proposition 4.2.

Now, it remains to find a way to see if a space satisfies property $(\mathrm{P})$ or not. We do not give a general condition here, but we prove that it is the case for the most classical spaces, endowed with their canonical filtration. More precisely:

Proposition 4.4. Let $\Omega$ be $\mathcal{C}\left(\mathbb{R}_{+}, \mathbb{R}^{d}\right)$, the space of continuous functions from $\mathbb{R}_{+}$to $\mathbb{R}^{d}$, or $\mathcal{D}\left(\mathbb{R}_{+}, \mathbb{R}^{d}\right)$, the space of càdlàg functions from $\mathbb{R}_{+}$to $\mathbb{R}^{d}$ (for some $d \geq 1$ ). For $t \geq 0$, define $\left(\mathcal{F}_{t}\right)_{t \geq 0}$ as the natural filtration of the canonical process $Y$, and $\mathcal{F}=\bigvee_{t \geq 0} \mathcal{F}_{t}$. Then $\left(\Omega, \mathcal{F},\left(\mathcal{F}_{t}\right)_{t \geq 0}\right)$ satisfies property $(P)$.

Proof. Let us prove this result for càdlàg functions (for continuous functions, the result is similar and is proved in [10]). For all $t \geq 0, \mathcal{F}_{t}$ is generated by the variables $Y_{r t}$, for $r$, rational, in [0,1], hence, it is countably generated. For the second property, one can take for $\Omega_{t}$, the set of càdlàg functions from $[0, t]$ to $\mathbb{R}^{d}$, and for $\pi_{t}$, the restriction to the interval $[0, t]$. The space $\Omega_{t}$ is Polish if one endows it with the Skorokhod metric. 
Moreover, its Borel $\sigma$-algebra is equal to the $\sigma$-algebra generated by the coordinates, a result from which one easily deduces the properties of $\pi_{t}$ which need to be satisfied. The third property is easy to check: let us suppose that $\left(\omega_{n}\right)_{n \geq 0}$ is a sequence of elements of $\Omega$, such that for all $N \geq 0$,

$$
\bigcap_{n=0}^{N} A_{n}\left(\omega_{n}\right) \neq \emptyset
$$

where $A_{n}\left(\omega_{n}\right)$ is the intersection of the sets in $\mathcal{F}_{n}$ containing $\omega_{n}$. Here, $A_{n}\left(\omega_{n}\right)$ is the set of functions $\omega^{\prime}$ which coincide with $\omega_{n}$ on $[0, n]$. Moreover, for $n \leq n^{\prime}$, integers, the intersection of $A_{n}\left(\omega_{n}\right)$ and $A_{n^{\prime}}\left(\omega_{n^{\prime}}\right)$ is not empty, and then $\omega_{n}$ and $\omega_{n^{\prime}}$ coincide on $[0, n]$. Therefore, there exists a càdlàg function $\omega$ which coincides with $\omega_{n}$ on $[0, n]$, for all $n$, which implies:

$$
\bigcap_{n=0}^{\infty} A_{n}\left(\omega_{n}\right) \neq \emptyset .
$$

Remark 4.5. It is easily seen that the conditions of Proposition 4.2 are not satisfied by the space $\mathcal{C}([0,1], \mathbb{R})$ endowed with the filtration generated by the canonical process; an explicit counter example is provided in [7].

We have now some examples of filtered probability spaces for which the extension of measures are always possible. However, these spaces do not possess any property of completion, and their filtration is not rightcontinuous. That is why we need to check that, at least in many interesting cases, this theorem of extension is still available after taking the natural augmentation. Before giving the corresponding statement, let us give a simple result, proving that many properties of stochastic processes are not changed by the natural augmentation of the underlying filtration.

Proposition 4.6. Let $\left(\Omega, \mathcal{F},\left(\mathcal{F}_{t}\right)_{t \geq 0}, \mathbb{P}\right)$ be a filtered probability space, and $\left(\Omega, \widetilde{\mathcal{F}},\left(\widetilde{\mathcal{F}}_{t}\right)_{t \geq 0}, \widetilde{\mathbb{P}}\right)$ its natural augmentation. Let $X$ be an $\mathcal{F}$-measurable random variable, integrable with respect to $\mathbb{P}$. Then $X$ is also integrable with respect to $\widetilde{\mathbb{P}}$, and

Moreover, for all $t \geq 0$

$$
\mathbb{E}_{\widetilde{\mathbb{P}}}[X]=\mathbb{E}_{\mathbb{P}}[X]
$$

$$
\mathbb{E}_{\widetilde{\mathbb{P}}}\left[X \mid \widetilde{\mathcal{F}}_{t}\right]=\mathbb{E}_{\mathbb{P}}\left[X \mid \mathcal{F}_{t}\right],
$$

$\widetilde{\mathbb{P}}$-almost surely.

Proof. For all sets $\Lambda \in \mathcal{F}$, one has

One deduces that

$$
\widetilde{\mathbb{P}}[\Lambda]=\mathbb{P}[\Lambda] .
$$

$$
\mathbb{E}_{\widetilde{\mathbb{P}}}[X]=\mathbb{E}_{\mathbb{P}}[X]
$$

for every nonnegative, $\mathcal{F}$-measurable random variable $X$, and then for every integrable variable $X$. Let $t \geq 0$ and let $\Lambda_{t}$ be an event in $\widetilde{\mathcal{F}}_{t}$. There exists an event $\Lambda_{t}^{\prime} \in \mathcal{F}_{t}$ such that its symmetric difference with $\Lambda_{t}$ is $\sigma$-negligible. One then has

$$
\begin{aligned}
\mathbb{E}_{\widetilde{\mathbb{P}}}\left[X \mathbb{1}_{\Lambda_{t}}\right]=\mathbb{E}_{\widetilde{\mathbb{P}}}\left[X \mathbb{1}_{\Lambda_{t}^{\prime}}\right] & =\mathbb{E}_{\mathbb{P}}\left[X \mathbb{1}_{\Lambda_{t}^{\prime}}\right]=\mathbb{E}_{\mathbb{P}}\left[\mathbb{E}_{\mathbb{P}}\left[X \mid \mathcal{F}_{t}\right] \mathbb{1}_{\Lambda_{t}^{\prime}}\right] \\
& =\mathbb{E}_{\widetilde{\mathbb{P}}}\left[\mathbb{E}_{\mathbb{P}}\left[X \mid \mathcal{F}_{t}\right] \mathbb{1}_{\Lambda_{t}^{\prime}}\right]=\mathbb{E}_{\widetilde{\mathbb{P}}}\left[\mathbb{E}_{\mathbb{P}}\left[X \mid \mathcal{F}_{t}\right] \mathbb{1}_{\Lambda_{t}}\right],
\end{aligned}
$$

which proves Proposition 4.6.

Proposition 4.6 has, in particular, the following consequence:

Corollary 4.7. Let $\left(\Omega, \mathcal{F},\left(\mathcal{F}_{t}\right)_{t \geq 0}, \mathbb{P}\right)$ be a filtered probability space, and $\left(\Omega, \widetilde{\mathcal{F}},\left(\widetilde{\mathcal{F}}_{t}\right)_{t \geq 0}, \widetilde{\mathbb{P}}\right)$ its natural augmentation. Then if $\left(X_{t}\right)_{t \geq 0}$ is a (sub)martingale with respect to the filtration $\left(\mathcal{F}_{t}\right)_{t \geq 0}$ and the probability $\mathbb{P}$, then it is also a (sub) martingale with respect to $\left(\widetilde{\mathcal{F}}_{t}\right)_{t \geq 0}$ and $\widetilde{\mathbb{P}}$. 
Proof. Let us suppose that $\left(X_{t}\right)_{t \geq 0}$ is a submartingale with respect to $\left(\mathcal{F}_{t}\right)_{t \geq 0}$ and $\mathbb{P}$. By Proposition 4.6, for all $t \geq 0, X_{t}$ is integrable with respect to $\widetilde{\mathbb{P}}$, and for $s \leq t$ :

$$
\mathbb{E}_{\tilde{\mathbb{P}}}\left[X_{t} \mid \widetilde{\mathcal{F}}_{s}\right]=\mathbb{E}_{\mathbb{P}}\left[X_{t} \mid \mathcal{F}_{s}\right] \geq X_{s}
$$

which implies that $\left(X_{t}\right)_{t \geq 0}$ is a submartingale with respect to $\left(\widetilde{\mathcal{F}}_{t}\right)_{t \geq 0}$ and $\widetilde{\mathbb{P}}$. The proof for martingales is exactly similar.

From Corollary 4.7, one deduces:

Corollary 4.8. Let $\left(\Omega, \mathcal{F},\left(\mathcal{F}_{t}\right)_{t \geq 0}, \mathbb{P}\right)$ be a filtered probability space, and $\left(\Omega, \widetilde{\mathcal{F}},\left(\widetilde{\mathcal{F}}_{t}\right)_{t \geq 0}, \widetilde{\mathbb{P}}\right)$ its natural augmentation. Then if $\left(X_{t}\right)_{t \geq 0}$ is a standard Brownian motion with respect to the filtration $\left(\mathcal{F}_{t}\right)_{t \geq 0}$ and the probability $\mathbb{P}$, then it is also a Brownian motion with respect to $\left(\widetilde{\mathcal{F}}_{t}\right)_{t \geq 0}$ and $\widetilde{\mathbb{P}}$.

Proof. We apply Corollary 4.7 to $\left(X_{t}\right)_{t \geq 0}$ and $\left(X_{t}^{2}-t\right)_{t \geq 0}$, and by continuity of $\left(X_{t}\right)_{t \geq 0}$, we are done.

Note that the results above are still available under usual augmentation, instead of natural augmentation (recall that usual augmentation is obtained by putting all the $\mathbb{P}$-negligible sets in $\mathcal{F}$ and $\mathcal{F}_{t}$ for all $t \geq 0$, and by completing the probability $\mathbb{P}$ ). However, in order to conserve the extension of coherent families of probabilities, one really needs to consider the natural augmentation instead of the usual augmentation. The precise statement is the following:

Proposition 4.9. Let $\left(\Omega, \mathcal{F},\left(\mathcal{F}_{t}\right)_{t \geq 0}, \mathbb{P}\right)$ be a filtered probability space, and $\left(\Omega, \widetilde{\mathcal{F}},\left(\widetilde{\mathcal{F}}_{t}\right)_{t \geq 0}, \widetilde{\mathbb{P}}\right)$ its natural augmentation. We assume that for all coherent families of probability measures $\left(\mathbb{Q}_{t}\right)_{t \geq 0}$, such that $\mathbb{Q}_{t}$ is defined on $\mathcal{F}_{t}$ for all $t \geq 0$, there exists a unique probability measure $\mathbb{Q}$ on $\mathcal{F}$ which coincides with $\mathbb{Q}_{t}$ on $\mathcal{F}_{t}$ for all $t \geq 0$. Then, for all coherent families of probability measures $\left(\widetilde{\mathbb{Q}}_{t}\right)_{t \geq 0}$, such that $\widetilde{\mathbb{Q}}_{t}$ is defined on $\widetilde{\mathcal{F}}_{t}$, and is absolutely continuous with respect to the restriction of $\widetilde{\mathbb{P}}$ to $\widetilde{\mathcal{F}}_{t}$, there exists a unique probability measure $\widetilde{\mathbb{Q}}$ on $\widetilde{\mathcal{F}}$ which coincides with $\widetilde{\mathbb{Q}}_{t}$ on $\widetilde{\mathcal{F}}_{t}$ for all $t \geq 0$.

Proof. Let $\left(\widetilde{\mathbb{Q}}_{t}\right)_{t \geq 0}$ be a coherent family of probability measures such that $\widetilde{\mathbb{Q}}_{t}$ is defined on $\widetilde{\mathcal{F}}_{t}$ and is absolutely continuous with respect to the restriction of $\widetilde{\mathbb{P}}$ to $\widetilde{\mathcal{F}}_{t}$. For all $t \geq 0$, one can consider the restriction $\mathbb{Q}_{t}$ of $\widetilde{\mathbb{Q}}_{t}$ to $\mathcal{F}_{t}$, and the family $\left(\mathbb{Q}_{t}\right)_{t \geq 0}$ is coherent. Indeed, for all $s \in[0, t]$ and for all events $\Lambda_{s} \in \mathcal{F}_{s}$, one has:

$$
\mathbb{Q}_{t}\left[\Lambda_{s}\right]=\widetilde{\mathbb{Q}}_{t}\left[\Lambda_{s}\right]=\widetilde{\mathbb{Q}}_{s}\left[\Lambda_{s}\right]=\mathbb{Q}_{s}\left[\Lambda_{s}\right]
$$

Therefore there exists a unique probability measure $\mathbb{Q}$ on $\mathcal{F}$ such that for all $t \geq 0$, the restriction of $\mathbb{Q}$ to $\mathcal{F}_{t}$ is $\mathbb{Q}_{t}$. Now, let $\left(\Omega, \mathcal{F}^{\prime},\left(\mathcal{F}_{t}^{\prime}\right)_{t \geq 0}, \mathbb{Q}^{\prime}\right)$ be the natural augmentation of $\left(\Omega, \mathcal{F},\left(\mathcal{F}_{t}\right)_{t \geq 0}, \mathbb{Q}\right)$. If an event $A$ is $\sigma$-negligible with respect to $\left(\Omega, \mathcal{F},\left(\mathcal{F}_{t}\right)_{t \geq 0}, \mathbb{P}\right)$, there exists $\left(B_{n}\right)_{n \geq 0}$, such that

$$
A \subset \bigcup_{n \geq 0} B_{n},
$$

and for all $n \geq 0, B_{n} \in \mathcal{F}_{n}$ and $\mathbb{P}\left[B_{n}\right]=0$. Since by assumption, $\widetilde{\mathbb{Q}}_{n}$ is absolutely continuous with respect to the restriction of $\widetilde{\mathbb{P}}$ to $\widetilde{\mathcal{F}}_{n}$ :

$$
\mathbb{Q}\left[B_{n}\right]=\mathbb{Q}_{n}\left[B_{n}\right]=\widetilde{\mathbb{Q}}_{n}\left[B_{n}\right]=\widetilde{\mathbb{P}}\left[B_{n}\right]=\mathbb{P}\left[B_{n}\right]=0 .
$$

One deduces that $A$ is $\sigma$-negligible with respect to $\left(\Omega, \mathcal{F},\left(\mathcal{F}_{t}\right)_{t \geq 0}, \mathbb{Q}\right)$. In other words, all the $\sigma$-negligible sets of $\left(\Omega, \mathcal{F},\left(\mathcal{F}_{t}\right)_{t \geq 0}, \mathbb{P}\right)$ are also $\sigma$-negligible with respect to $\left(\Omega, \mathcal{F},\left(\mathcal{F}_{t}\right)_{t \geq 0}, \mathbb{Q}\right)$, which implies that $\mathcal{F}^{\prime}$ contains $\widetilde{\mathcal{F}}$. Therefore, one can define the probability measure $\widetilde{\mathbb{Q}}$ as the restriction of $\mathbb{Q}^{\prime}$ to the $\sigma$-algebra $\widetilde{\mathcal{F}}$ : it remains to check that this measure satisfies Proposition 4.9. Indeed, let $\Lambda_{t}$ be an event in $\widetilde{\mathcal{F}}_{t}$, for some $t \geq 0$. There exists 
$\Lambda_{t}^{\prime} \in \mathcal{F}_{t+}$ such that its symmetric difference with $\Lambda_{t}$ is $\sigma$-negligible with respect to $\left(\Omega, \mathcal{F},\left(\mathcal{F}_{t}\right)_{t \geq 0}, \mathbb{P}\right)$, and then also with respect to $\left(\Omega, \mathcal{F},\left(\mathcal{F}_{t}\right)_{t \geq 0}, \mathbb{Q}\right)$. One deduces that

$$
\begin{aligned}
\widetilde{\mathbb{Q}}\left[\Lambda_{t}\right]=\mathbb{Q}^{\prime}\left[\Lambda_{t}\right] & =\mathbb{Q}\left[\Lambda_{t}^{\prime}\right]=\mathbb{Q}_{t+1}\left[\Lambda_{t}^{\prime}\right] \\
& =\widetilde{\mathbb{Q}}_{t+1}\left[\Lambda_{t}^{\prime}\right]=\widetilde{\mathbb{Q}}_{t+1}\left[\Lambda_{t}\right] \\
& =\widetilde{\mathbb{Q}}_{t}\left[\Lambda_{t}\right] .
\end{aligned}
$$

Here the equality

$$
\widetilde{\mathbb{Q}}_{t+1}\left[\Lambda_{t}^{\prime}\right]=\widetilde{\mathbb{Q}}_{t+1}\left[\Lambda_{t}\right]
$$

is due to the fact that $\Lambda_{t}^{\prime}$ and $\Lambda_{t}$ are both in $\widetilde{\mathcal{F}}_{t+1}$, their symmetric difference is $\sigma$-negligible with respect to $\left(\Omega, \mathcal{F},\left(\mathcal{F}_{t}\right)_{t \geq 0}, \mathbb{P}\right)$, and $\widetilde{\mathbb{Q}}_{t+1}$ is absolutely continuous with respect to the restriction of $\widetilde{\mathbb{P}}$ to $\widetilde{\mathcal{F}}_{t+1}$. The uniqueness of $\widetilde{\mathbb{Q}}$ is proved as follows: its restriction $\mathbb{Q}$ to $\mathcal{F}$ is uniquely determined, since it has to coincide with $\mathbb{Q}_{t}$ on $\mathcal{F}_{t}$ (recall that $\mathbb{Q}_{t}$ is the restriction of $\widetilde{\mathbb{Q}}_{t}$ to $\mathcal{F}_{t}$ ). Now, for all events $\Lambda \in \widetilde{\mathcal{F}}$, there exists $\Lambda^{\prime} \in \mathcal{F}$ such that the symmetric difference of $\Lambda$ and $\Lambda^{\prime}$ is $\sigma$-negligible with respect to $\left(\Omega, \mathcal{F},\left(\mathcal{F}_{t}\right)_{t \geq 0}, \mathbb{P}\right)$, and then with respect to $\left(\Omega, \mathcal{F},\left(\mathcal{F}_{t}\right)_{t>0}, \mathbb{Q}\right)$. One deduces that $\left(\Lambda \backslash \Lambda^{\prime}\right) \cup\left(\Lambda^{\prime} \backslash \Lambda\right)$ is included in a set $B \in \mathcal{F}$ such that $\mathbb{Q}[B]=0$, and then

$$
\widetilde{\mathbb{Q}}\left[\left(\Lambda \backslash \Lambda^{\prime}\right) \cup\left(\Lambda^{\prime} \backslash \Lambda\right)\right] \leq \widetilde{\mathbb{Q}}[B]=\mathbb{Q}[B]=0,
$$

which implies

and $\widetilde{\mathbb{Q}}[\Lambda]$ is uniquely determined.

$$
\widetilde{\mathbb{Q}}[\Lambda]=\widetilde{\mathbb{Q}}\left[\Lambda^{\prime}\right]=\mathbb{Q}\left[\Lambda^{\prime}\right]
$$

One immediately deduces, from Propositions 4.2 and 4.9, the following:

Corollary 4.10. Let $\left(\Omega, \mathcal{F},\left(\mathcal{F}_{t}\right)_{t \geq 0}, \mathbb{P}\right)$ be the natural augmentation of a filtered probability space satisfying the property $(P)$. Then if $\left(\mathbb{Q}_{t}\right)_{t \geq 0}$ is a coherent family of probability measures, $\mathbb{Q}_{t}$ defined on $\mathcal{F}_{t}$, and absolutely continuous with respect to the restriction of $\mathbb{P}$ to $\mathcal{F}_{t}$, there exists a unique probability measure $\mathbb{Q}$ on $\mathcal{F}$ which coincides with $\mathbb{Q}_{t}$ on $\mathcal{F}_{t}$, for all $t \geq 0$.

We observe that if $\left(\Omega, \mathcal{F},\left(\mathcal{F}_{t}\right)_{t \geq 0}\right)$ satisfies the property $(\mathrm{P})$, then $\mathcal{F}$ is the $\sigma$-algebra generated by $\mathcal{F}_{t}, t \geq 0$. This property is clearly preserved when one takes the natural augmentation.

Acknowledgements. We would like to thank F. Delbaen for all his enlightening comments during the preparation of this paper, and H. Föllmer for very helpful discussions. We also wish to thank R. van Handel for bringing to our attention the book by K. Bichteler where the new augmentation we suggest was first proposed.

\section{REFERENCES}

[1] K. Bichteler, Stochastic integration with jumps. Cambridge University Press (2002).

[2] K. Ciesielski, How good is Lebesgue measure? Math. Intell. 11 (1989) 54-58.

[3] C. Dellacherie and P.-A. Meyer, Un nouveau théorème de projection et de section, in Séminaire de Probabilités XXVI. Lecture Notes in Math. 1526. Springer (1975) 239-245.

[4] C. Dellacherie and P.-A. Meyer, Probabilités et potentiel, Volume 1. Hermann, Paris (1976).

[5] C. Dellacherie and P.-A. Meyer, Probabilités et potentiel, Volume 2. Hermann, Paris (1980).

[6] H. Föllmer, The exit measure of a supermartingale. Z. Wahrscheinlichkeitstheorie verw. Geb. 21 (1972) $154-166$.

[7] H. Föllmer and P. Imkeller, Anticipation cancelled by a Girsanov transformation: a paradox on Wiener space. Ann. Inst. H. Poincaré 29 (1993) 569-586.

[8] J. Jacod and A. Shiryaev, Limit theorems for stochastic processes. 2nd edition. Springer (2003).

[9] K.R. Parthasarathy, Probability measures on metric spaces. Academic Press, New York (1967).

[10] D.-W. Stroock and S.-R.-S. Varadhan, Multidimensional diffusion processes, Classics in Mathematics. Springer-Verlag, Berlin (2006). Reprint of the 1997 edition. 